\title{
Smooth operators in the commutant of a contraction
}

\author{
by \\ Pascale Vitse (Québec)
}

\begin{abstract}
For a completely non-unitary contraction $T$, some necessary (and, in certain cases, sufficient) conditions are found for the range of the $H^{\infty}$ calculus, $H^{\infty}(T)$, and the commutant, $\{T\}^{\prime}$, to contain non-zero compact operators, and for the finite rank operators of $\{T\}^{\prime}$ to be dense in the set of compact operators of $\{T\}^{\prime}$. A sufficient condition is given for $\{T\}^{\prime}$ to contain non-zero operators from the Schatten-von Neumann classes $S_{p}$.
\end{abstract}

1. Introduction. For a given Hilbert space contraction $T$, we study how "smooth" (compact, etc.) operators in the commutant $\{T\}^{\prime}=\{A$ : $A T=T A\}$ can be. The problem arises in several applications in control theory, vector-valued Hankel operators or the theory of model operators. Here it is treated in the framework of the Sz.-Nagy-Foias functional model and some answers are proposed in the language of the characteristic function $\Theta_{T}$ of the contraction $T$.

Let $\mathcal{H}$ be a separable Hilbert space, and $L(\mathcal{H})$ the space of bounded linear operators on $\mathcal{H}$. Let $\mathcal{F}$ and $S_{\infty}$ denote the subspaces of $L(\mathcal{H})$ consisting respectively of the finite-rank and compact operators. Let $S_{p}(0<p<\infty)$ denote the Schatten-von Neumann class consisting of the compact operators on $\mathcal{H}$ for which the sequence of singular numbers belongs to $l^{p}$. Only completely non-unitary (c.n.u.) contractions are considered. The questions studied in this paper are the following. Let $T$ be a c.n.u. contraction on $\mathcal{H}$. When does $\{T\}^{\prime}$ contain non-zero finite-rank operators, non-zero compact operators, or non-zero operators from the class $S_{p}$ ? When is $\{T\}^{\prime} \cap \mathcal{F}$ dense in $\{T\}^{\prime} \cap S_{\infty}$ ? Some of these questions are also considered for the range space of the functional calculus $H^{\infty}(T)=\left\{\varphi(T): \varphi \in H^{\infty}\right\}$ instead of $\{T\}^{\prime}$. Clearly, $H^{\infty}(T) \subset\{T\}^{\prime}$.

For contractions of some specific classes, several facts are known about the above problems. For instance, for operators from the class $C_{0}$ (that is, for c.n.u. contractions $T$ for which the $H^{\infty}$ calculus $\varphi \mapsto \varphi(T)$ has a non-zero kernel) the following is proved:

2000 Mathematics Subject Classification: 47A45, 47A60, 47B10, 47A20. 
(i) (Sz.-Nagy, [SN74]) Always, $\{T\}^{\prime} \cap S_{\infty} \neq\{0\}$, but it may happen that $H^{\infty}(T) \cap S_{\infty}=\{0\}$.

(ii) (Nordgren, [Nor75]) If $I-T^{*} T \in S_{\infty}$ then $H^{\infty}(T) \cap S_{\infty} \neq\{0\}$; and moreover, there exists a sequence $\left(\varphi_{n}\right)_{n \geq 1} \subset H^{\infty}$ such that $\left\|\varphi_{n}\right\|_{\infty} \leq 1$, $\varphi_{n}(T) \in S_{\infty}$ for $n \geq 1$, and (WOT)- $\lim \varphi_{n}(T)=I$. Here $I$ denotes the identity operator and WOT stands for Weak Operator Topology.

Before answering the above questions, we recall some elements of the Sz.-Nagy-Foiaş model. First, it is worth mentioning that every Hilbert space contraction is an orthogonal sum of a unitary operator and a c.n.u. contraction, and that for the unitary part the questions related to the commutant and functional calculus can be easily answered via the von Neumann spectral theorem. In what follows, $\mathbb{T}$ is the unit circle of the complex plane, $\mathbb{T}=\{z \in \mathbb{C}:|z|=1\}$, and $\mathbb{D}$ is the unit disc, $\mathbb{D}=\{z \in \mathbb{C}:|z|<1\}$.

For a given c.n.u. contraction $T$, the main object of the functional model approach is the characteristic function $\Theta=\Theta_{T}$ defined by

$$
\Theta(z)=\left.\left[-T+z D_{T^{*}}\left(I-z T^{*}\right)^{-1} D_{T}\right]\right|_{\mathcal{D}_{T}}, \quad z \in \mathbb{D} .
$$

Here $D_{T}=\left(I-T^{*} T\right)^{1 / 2}$ and $D_{T^{*}}=\left(I-T T^{*}\right)^{1 / 2}$ are the defect operators of $T, \mathcal{D}_{T}=\operatorname{clos} D_{T} \mathcal{H}$ and $\mathcal{D}_{T^{*}}=\operatorname{clos} D_{T^{*}} \mathcal{H}$ are the defect spaces of $T$. In fact, $\Theta$ is an analytic contractive-valued $(\|\Theta(z)\| \leq 1, z \in \mathbb{D})$ function from $\mathcal{D}_{T}$ to $\mathcal{D}_{T^{*}}$; in particular, $\Theta$ belongs to $H^{\infty}\left(L\left(\mathcal{D}_{T}, \mathcal{D}_{T^{*}}\right)\right)$. The main theorem of the model theory says that $T$ is unitarily equivalent to the model operator $M_{\Theta}$ defined on the model space $K_{\Theta}$ by the following formulas:

$$
\begin{aligned}
& K_{\Theta}=\left(\begin{array}{c}
H^{2}\left(\mathcal{D}_{T^{*}}\right) \\
\operatorname{clos} \Delta L^{2}\left(\mathcal{D}_{T}\right)
\end{array}\right) \ominus\left(\begin{array}{c}
\Theta \\
\Delta
\end{array}\right) H^{2}\left(\mathcal{D}_{T}\right), \\
& M_{\Theta} \in L\left(K_{\Theta}\right), \quad M_{\Theta} f=P_{\Theta} z f, \quad f \in K_{\Theta} .
\end{aligned}
$$

Here, for a Hilbert space $E, L^{2}(E)$ denotes the Bochner-Lebesgue space of square integrable $E$-valued strongly measurable functions on $\mathbb{T} ; H^{2}(E)$ denotes the Hardy space of $E$-valued analytic functions, $H^{2}(E) \subset L^{2}(E)$; $\Delta$ is the defect operator of $\Theta$ defined by $\Delta(\xi)=\left(I-\Theta(\xi)^{*} \Theta(\xi)\right)^{1 / 2}$, which for almost every $\xi \in \mathbb{T}$ is a bounded $L\left(\mathcal{D}_{T}\right)$-valued function on $\mathbb{T}$, that is, $\Delta \in L^{\infty}\left(L\left(\mathcal{D}_{T}\right)\right)$; and, finally, $P_{\Theta}$ denotes the orthogonal projection from $L^{2}\left(\mathcal{D}_{T^{*}} \oplus \mathcal{D}_{T}\right)$ onto $K_{\Theta}$. More details on the model operators are given in Section 1 below and a complete exposition can be found in the book [SNF67]. In principle, the notation here follows that of this book.

Some of our answers are valid for the general situation of an arbitrary c.n.u. contraction $T$, others for particular classes of contractions, mainly in the case when the characteristic function has a scalar multiple. The following theorems are the main results of this paper. $T$ is always a c.n.u. contraction. Inner and outer functions and the different classes of contractions are defined in Subsection 2.2 below. 
Theorem. Assume that $H^{\infty}(T) \cap S_{\infty} \neq\{0\}$. Then $\Theta_{T}$ is a two-sided inner function. In the case when $T$ is an (SM)-contraction and $I-T^{*} T \in S_{\infty}$ the converse is also true.

This theorem reflects a feeling that the outer factor of $\Theta_{T}$ corresponds to a part of the operator similar in some sense to a unitary one. It is well known that, when a unitary operator has absolutely continuous spectrum, the only compact operator in the commutant is zero.

The following result means that only the trace-class smoothness of $D_{T}^{2}$ can guarantee the existence of non-zero compact $H^{\infty}$ functions of $T$. Here $\sigma(T)$ denotes the spectrum of $T$, and $\sigma_{\mathrm{p}}(T)$ the point spectrum of $T$, that is, the set of eigenvalues of $T$.

TheOREM. Let $\mathfrak{S} \subset S_{\infty}$ be a symmetrically normed ideal of $L(\mathcal{H})$. The following are equivalent:

(i) For every c.n.u. contraction $T \in C_{00}$ such that $\mathbb{D} \backslash \sigma(T)$ is non-empty and $I-T^{*} T \in \mathfrak{S}$, we have

$$
H^{\infty}(T) \cap S_{\infty} \neq\{0\} .
$$

(ii) $\mathfrak{S}=S_{1}$.

Passing to the commutant $\{T\}^{\prime}$ we first prove that if $I-T^{*} T \in S_{\infty}$ and $\mathbb{D} \backslash \sigma(T) \neq \emptyset$, then $\{T\}^{\prime} \cap \mathcal{F} \neq\{0\}$ if and only if $\mathbb{D} \cap \sigma(T)$ is non-empty. Next, we obtain the following criterion.

Theorem. Let $T \in L(\mathcal{H})$ be a c.n.u. contraction. If $T \in C_{1} \cup C_{.1}$ (equivalently, $\Theta_{T}$ is either outer or $*$-outer) then $\{T\}^{\prime} \cap S_{\infty}=\{0\}$. If , moreover, $T \in(\mathrm{SM})$ and $I-T^{*} T \in S_{\infty}$, then the converse is also true, and in fact, $\{T\}^{\prime} \cap S_{\infty}=\{0\}$ implies that $T \in C_{11}$ (equivalently, $\Theta_{T}$ is two-sided outer).

In particular, if $\Theta \in H^{\infty}$ is a non-zero contractive-valued (scalar) function, then $\left\{M_{\Theta}\right\}^{\prime} \cap S_{\infty}=\{0\}$ if and only if $\Theta$ is outer, and $H^{\infty}\left(M_{\Theta}\right) \cap S_{\infty}$ $=\{0\}$ if and only if $\Theta$ is not inner.

Next, we pass to the question of the density of $\{T\}^{\prime} \cap \mathcal{F}$ in $\{T\}^{\prime} \cap S_{\infty}$ for $T \in(\mathrm{SM})$ such that $I-T^{*} T \in S_{\infty}$. It can be formulated in terms of the restriction $T_{0}$ of $T$ to the invariant subspace $\mathcal{H}_{0}(T)=\left\{x \in \mathcal{H}:\left\|T^{n} x\right\| \rightarrow 0\right\}$. Then $T_{0}$ is a $C_{0}$-contraction and density holds if and only if $T_{0}$ is complete (equivalently $m_{T_{0}}$ is a Blaschke product). In this case we always have a linear approximation process, and $\mathcal{H}_{0}(T)$ coincides with $E_{T}=\operatorname{clos}(\bigcup\{X \mathcal{H}$ : $\left.\left.X \in\{T\}^{\prime} \cap S_{\infty}\right\}\right)$.

It is likely that $\mathcal{H}_{0}(T)=E_{T}$ for any (SM)-contraction with $I-T^{*} T \in S_{\infty}$. But at the moment we can only prove that $m_{T_{0}}=m_{T_{E}}$, where $T_{E}=\left.T\right|_{E_{T}}$.

The last result deals with operators in the Schatten-von Neumann ideals $S_{p}$. 
TheOREM. Let $T$ be an (SM)-contraction such that $I-T^{*} T \in S_{p}$ with $1 \leq p<\infty$. Then $\{T\}^{\prime} \cap S_{p} \neq\{0\}$ as soon as one of the following properties is satisfied:

(i) $\sigma_{\mathrm{p}}(T) \neq \emptyset$, or equivalently $\operatorname{ker} \Theta_{T}(\lambda) \neq\{0\}$ for some $\lambda \in \mathbb{D}$.

(ii) There exists a Beurling-Carleson set $\sigma \subset \mathbb{T}$ such that $H(\sigma) \neq\{0\}$, where $H(\sigma)$ stands for the maximal spectral subspace over $\sigma$ (see Section 5 for definitions).

Moreover if $T \in C_{0}$ then $H^{\infty}(T) \cap S_{p} \neq\{0\}$.

The techniques used for the proofs of the above results are mostly based on the Commutant Lifting Theorem (CLT for short), the cornerstone of the theory of model operators. Via the CLT, the problems are reduced to certain questions about vector-valued Hankel operators. In the case of twosided inner characteristic functions this reduction was known long ago (see [Nik86]). For a more general case, a new formula is established below to link compact operators in the commutant and Hankel operators (see Lemma 4.4). Then Muhly's and Peller's theories of smooth Hankel operators are used.

The paper is organized as follows. Section 2 contains necessary prerequisites on the Sz.-Nagy-Foias functional model. Section 3 is devoted to smooth operators in $H^{\infty}(T)$. Section 4 deals with compact and finite rank operators in $\{T\}^{\prime}$, and Section 5 is devoted to the Schatten-von Neumann classes $S_{p}$.

2. Some facts about the canonical model. Let $\Theta \in H^{\infty}\left(L\left(\mathcal{H}_{1}, \mathcal{H}_{2}\right)\right)$ be any contractive-valued function, where $\mathcal{H}_{1}, \mathcal{H}_{2}$ are two separable Hilbert spaces. Then $\Theta$ is called pure if $\|\Theta(0) x\|<\|x\|$ for all $x \in \mathcal{H}_{1}, x \neq 0$. For every contractive-valued $\Theta \in H^{\infty}\left(L\left(\mathcal{H}_{1}, \mathcal{H}_{2}\right)\right)$, there exists a unique pure contractive-valued function $\Theta^{0}$ and a constant unitary operator $U$ acting between certain subspaces of $\mathcal{H}_{1}$ and $\mathcal{H}_{2}$ respectively such that $\Theta(z)=$ $\Theta^{0}(z) \oplus U$. This $\Theta^{0}$ is called the pure part of $\Theta$. Let $\Theta \in H^{\infty}\left(L\left(\mathcal{H}_{1}, \mathcal{H}_{2}\right)\right)$ be a contractive-valued function, and $M_{\Theta}$ be defined as in Section 1. Then $M_{\Theta}$ is a c.n.u. contraction and the characteristic function of $M_{\Theta}$ coincides with the pure part of $\Theta$.

2.1. The commutant lifting theorem (CLT). Here $T$ is identified with $M_{\Theta}$. It is clear that for every $\varphi \in H^{\infty}$ the lifting formula $\varphi\left(M_{\Theta}\right)=\left.P_{\Theta} \varphi\right|_{K_{\Theta}}$ holds. As already mentioned, $H^{\infty}\left(M_{\Theta}\right) \subset\left\{M_{\Theta}\right\}^{\prime}$. It is known that $\left\{M_{\Theta}\right\}^{\prime}=$ $H^{\infty}\left(M_{\Theta}\right)$ when $\Theta$ is a scalar inner function [Sar67]. In general, this is not the case. The characterization of the c.n.u. contractions $T$ such that $H^{\infty}(T)=$ $\{T\}^{\prime}$ seems to be unknown and is a delicate problem. However the above lifting formula extends to operators from $\left\{M_{\Theta}\right\}^{\prime}$ thanks to the CLT due to Sz.-Nagy and Foiaş [SNF67]. Namely, $X \in\left\{M_{\Theta}\right\}^{\prime}$ if and only if there exists an operator $Y$ acting on $\left(\begin{array}{c}H^{2}\left(\mathcal{D}_{T^{*}}\right) \\ \operatorname{clos} \Delta L^{2}\left(\mathcal{D}_{T}\right)\end{array}\right)$ such that $X=\left.P_{\Theta} Y\right|_{K_{\Theta}}, Y z=z Y$, 
and

$$
Y\left(\begin{array}{c}
\Theta \\
\Delta
\end{array}\right) H^{2}\left(\mathcal{D}_{T}\right) \subset\left(\begin{array}{c}
\Theta \\
\Delta
\end{array}\right) H^{2}\left(\mathcal{D}_{T}\right) .
$$

Then $Y$ is called a lifting of $X$. The space $\left(\begin{array}{c}H^{2}\left(\mathcal{D}_{T^{*}}\right) \\ \operatorname{clos} \Delta L^{2}\left(\mathcal{D}_{T}\right)\end{array}\right)$ is actually the space of the minimal isometric dilation of $M_{\Theta}$.

Notice that in the case when $\Theta$ is a two-sided inner function (see below) the two lifting conditions are equivalent to $Y \in H^{\infty}\left(L\left(\mathcal{D}_{T^{*}}\right)\right)$ and $\Theta^{*} Y \Theta \in$ $H^{\infty}\left(L\left(\mathcal{D}_{T}\right)\right)$ and the CLT admits an alternative proof due to N. K. Nikolski [Nik86], making use of Hankel operators. Moreover, if $Y$ is a lifting of $X$, then $X=\left.\Theta H_{\Theta^{*} Y}\right|_{K_{\Theta}}$, as in this case $P_{\Theta}=\Theta P_{-} \Theta^{*}$.

It follows that a lifting $Y$ is an operator of multiplication by a block matrix function of the type

$$
Y=\left(\begin{array}{cc}
A_{1} & 0 \\
B_{1} & C_{1}
\end{array}\right)
$$

where

$A_{1} \in H^{\infty}\left(L\left(\mathcal{D}_{T^{*}}\right)\right), \quad B_{1} \in L^{\infty}\left(L\left(\mathcal{D}_{T^{*}}, \operatorname{clos} \Delta \mathcal{D}_{T}\right)\right), \quad C_{1} \in L^{\infty}\left(L\left(\operatorname{clos} \Delta \mathcal{D}_{T}\right)\right)$

are operator-valued functions satisfying the following relations:

$$
A_{1}(\xi) \Theta(\xi)=\Theta(\xi) A_{0}(\xi), \quad B_{1}(\xi) \Theta(\xi)+C_{1}(\xi) \Delta(\xi)=\Delta(\xi) A_{0}(\xi)
$$

a.e. on $\mathbb{T}$, for some $A_{0} \in H^{\infty}\left(L\left(\mathcal{D}_{T}\right)\right)$.

Any lifting of the zero operator is of the form $Y=\left(\begin{array}{cc}\Theta G & 0 \\ \Delta G & 0\end{array}\right)$ for some $G \in H^{\infty}\left(L\left(\mathcal{D}_{T^{*}}, \mathcal{D}_{T}\right)\right)$. Moreover,

$$
\begin{aligned}
\|X\| & =\inf \left\{\|Y\|_{\infty}: Y \text { a lifting of } X\right\} \\
& =\inf \left\{\left\|Y_{0}+\left(\begin{array}{cc}
\Theta G & 0 \\
\Delta G & 0
\end{array}\right)\right\|: G \in H^{\infty}\left(L\left(\mathcal{D}_{T^{*}}, \mathcal{D}_{T}\right)\right)\right\},
\end{aligned}
$$

where $Y_{0}$ is any lifting of $Y$; the infimum is always attained. Notice that a lifting of a function of $M_{\Theta}, X=\varphi\left(M_{\Theta}\right) \in\left\{M_{\Theta}\right\}^{\prime}, \varphi \in H^{\infty}$, corresponds to $B_{1}=0, A_{1}=\varphi I, C_{1}=\varphi I$.

Another parametrization of the liftings of the operators in $\left\{M_{\Theta}\right\}^{\prime}$ is

$$
Y=\left(\begin{array}{cc}
A_{*} & 0 \\
\Delta A \Theta^{*}+B \Delta_{*} & \Delta A \Delta-B \Theta
\end{array}\right),
$$

where $A \in H^{\infty}\left(L\left(\mathcal{D}_{T}\right)\right), A_{*} \in H^{\infty}\left(L\left(\mathcal{D}_{T^{*}}\right)\right)$ satisfy $\Theta A=A_{*} \Theta$, and $B \in$ $L^{\infty}\left(L\left(\Delta_{*} \mathcal{D}_{T^{*}}, \Delta \mathcal{D}_{T}\right)\right)$; see [NV98].

\subsection{Classes of contractions}

$C_{\alpha \beta}$ classes. Let $T$ be a c.n.u. contraction. Then $T$ is of class $C_{0}$. if $T^{n}$ tends SOT (Strong Operator Topology) to zero (i.e. $\lim _{n \rightarrow \infty}\left\|T^{n} x\right\|=0$ for every $x \in \mathcal{H}$ ), and $T$ is $C_{1}$. if $\left\|T^{n} x\right\|$ does not tend to 0 for every $x \neq 0$. For 
$\alpha, \beta=0,1$, the contraction $T$ is $C_{. \alpha}$ if $T^{*}$ is $C_{\alpha .}$, and $T$ is $C_{\alpha \beta}$ if it is both $C_{\alpha}$. and $C_{. \beta}$.

Let $\Theta$ be the characteristic function of $T$. Then $T$ is $C_{0}$. (resp. $C_{.0}$, $\left.C_{1 .}, C_{.1}\right)$ if $\Theta$ is $*$-inner (resp. inner, *-outer, outer). Recall that a function $F \in H^{\infty}\left(L\left(\mathcal{H}_{1}, \mathcal{H}_{2}\right)\right)$ is inner if the non-tangential limits on $\mathbb{T}$ are isometric almost everywhere. It is outer if $F H^{2}\left(\mathcal{H}_{1}\right)$ is dense in $H^{2}\left(\mathcal{H}_{2}\right)$, where $F$ is identified with the operator of multiplication by $F$. The function $F$ is $*$-inner (resp. *-outer) if $F^{\mathrm{t}}$ is inner (resp. outer), where $F^{\mathrm{t}}(z)=F(\bar{z})^{*}$. It is twosided inner (resp. two-sided outer) if $F$ is both inner and *-inner (resp. outer and $*$-outer $)$. Every function $F \in H^{\infty}\left(L\left(\mathcal{H}_{1}, \mathcal{H}_{2}\right)\right)$ admits a canonical innerouter factorization $F=F_{\text {inn }} F_{\text {out }}$ through an intermediate Hilbert space (and consequently, also a canonical $*$-outer- $*$-inner factorization).

Let $T \in L(\mathcal{H})$ be a contraction. Then $\mathcal{H}_{0}=\left\{x \in \mathcal{H}:\left\|T^{n} x\right\| \rightarrow 0\right\}$ is an invariant subspace of $T$ and the decomposition $\mathcal{H}=\mathcal{H}_{0} \oplus \mathcal{H}_{0}^{\perp}$ induces a triangular decomposition of $T$, which is called the $C_{0 .}-C_{1}$. decomposition of $T$ :

$$
\left(\begin{array}{cc}
T_{0} & * \\
0 & T_{1}^{\prime}
\end{array}\right), \quad T_{0} \in C_{0 .}, T_{1}^{\prime} \in C_{1 .}
$$

It is the only decomposition of $T$ satisfying (3). Applying the result to $T^{*}$, we find that $T$ admits a unique triangular decomposition of the form

$$
\left(\begin{array}{cc}
T_{1} & * \\
0 & T_{0}^{\prime}
\end{array}\right), \quad T_{1} \in C_{.1}, T_{0}^{\prime} \in C_{.0}
$$

where $T_{1}=\left.T\right|_{\mathcal{H}_{1}}$ and $\mathcal{H}_{1}=\left\{x \in \mathcal{H}: T^{* n} x \rightarrow 0\right\}^{\perp}$. It is called the $C_{.1^{-}} C_{.0}$ decomposition of $T$.

$C_{0}$-contractions. Let $T \in L(\mathcal{H})$ be a contraction. By definition $T \in C_{0}$ if $T$ is c.n.u. and there exists a function $u \in H^{\infty}, u \neq 0$, such that $u(T)=0$. For every $T \in C_{0}$, there exists a minimal (annihilating) function $m_{T}$ (unique within a unimodular constant); that is, $m_{T}$ is an inner function such that $m_{T}(T)=0$, and if $u(T)=0$ for some $u \in H^{\infty} \backslash\{0\}$, then $u / m_{T} \in H^{\infty}$ (see [SNF67, Proposition III.4.4]).

Recall that the spectrum $\sigma(\varphi)$ of a contractive-valued function $\varphi \in H^{\infty}$ is defined by

$$
\sigma(\varphi)=(\operatorname{clos}\{\lambda \in \mathbb{D}: \varphi(\lambda)=0\}) \cup \operatorname{supp}\left(\left.\Delta_{\varphi}\right|_{\mathbb{T}}\right) \cup \operatorname{supp}\left(\mu_{\varphi}^{\mathrm{s}}\right),
$$

where $\Delta_{\varphi}=\left(1-|\varphi|^{2}\right)^{1 / 2}$ and $\mu_{\varphi}^{\mathrm{s}}$ is the singular measure on $\mathbb{T}$ associated to $\varphi$ through its Nevanlinna-Riesz-Smirnov canonical factorization. Let $T \in$ $L(\mathcal{H})$ be a $C_{0}$-contraction with minimal function $m_{T}$. Then $\sigma(T)=\sigma\left(m_{T}\right)$ (see [SNF67, Theorem III.5.1]). In particular, $\sigma_{\mathrm{p}}(T)=\sigma\left(m_{T}\right) \cap \mathbb{D}=\sigma(T) \cap \mathbb{D}$ consists of the zeros of $m_{T}$, thus $\sigma(T) \cap \mathbb{D}$ is a (possibly empty) Blaschke sequence. Recall also that a $C_{0}$-contraction $T$ is complete (i.e. $\mathcal{H}$ is spanned 
by the generalized eigenvectors of $T$, see Subsection 4.3) if and only if $m_{T}$ is a Blaschke product.

Scalar multiples and (SM)-contractions. A function $\Theta \in H^{\infty}\left(L\left(\mathcal{H}_{1}, \mathcal{H}_{2}\right)\right)$ has a scalar multiple $\delta \in H^{\infty}, \delta \neq 0$, if there exists $\Omega \in H^{\infty}\left(L\left(\mathcal{H}_{2}, \mathcal{H}_{1}\right)\right)$ such that $\Omega \Theta=\delta I$ and $\Theta \Omega=\delta I$. Obviously, we then have $\operatorname{dim} \mathcal{H}_{1}=\operatorname{dim} \mathcal{H}_{2}$ and $\Theta$ is invertible at every point $z \in \mathbb{D}$ such that $\delta(z) \neq 0$, and then

$$
\Theta(z)^{-1}=\frac{1}{\delta(z)} \Omega(z) .
$$

In particular, $\Theta^{-1}$ is a meromorphic function in $\mathbb{D}$.

Let $\Theta \in H^{\infty}\left(L\left(\mathcal{H}_{1}, \mathcal{H}_{2}\right)\right)$ be a contractive-valued function having a scalar multiple $\delta$. If $\Theta=\Theta_{\text {inn }} \Theta_{\text {out }}$ is the canonical inner-outer factorization of $\Theta$, then $\Theta_{\text {inn }}$ and $\Theta_{\text {out }}$ admit respectively $\delta_{\text {inn }}$ and $\delta_{\text {out }}$ as scalar multiples, where $\delta_{\text {inn }}$ and $\delta_{\text {out }}$ are the inner and outer parts of $\delta$. In particular, $\Theta_{\text {inn }}$ is two-sided inner and $\Theta_{\text {out }}$ is two-sided outer. Similarly $\Theta$ admits an outerinner factorization, $\Theta=\Theta_{\text {out }}^{\prime} \Theta_{\text {inn }}^{\prime}$, where $\delta_{\text {out }}$ and $\delta_{\text {inn }}$ are scalar multiples of $\Theta_{\text {out }}^{\prime}$ and $\Theta_{\text {inn }}^{\prime}$ (see [SNF67, Theorem V.6.2]).

Let $T \in L(\mathcal{H})$ be a c.n.u. contraction. We write $T \in(\mathrm{SM})$ if $\Theta_{T}$ has a scalar multiple. If $T$ has $\delta$ as a scalar multiple, then $\sigma_{\mathrm{p}}(T)=\sigma(T) \cap \mathbb{D} \subset$ $\{\lambda \in \mathbb{D}: \delta(\lambda)=0\}$, and thus $\sigma(T) \cap \mathbb{D}$ is a (possibly empty) Blaschke sequence; in particular $\mathbb{D} \backslash \sigma(T) \neq \emptyset$.

For $T \in(\mathrm{SM})$, the components $T_{0}, T_{1}^{\prime}$ of the $C_{0}-C_{1}$. decomposition (3) are (SM)-contractions and the $C_{0 .}-C_{1}$. decomposition is in fact a $C_{0^{-}} C_{11}$ decomposition. If $\delta$ is a scalar multiple of $\Theta_{T}$, then $\delta_{\text {inn }}$ and $\delta_{\text {out }}$ are scalar multiples of $T_{0}$ and $T_{1}^{\prime}$. Then $m_{T_{0}}$ is the minimal scalar multiple of $T_{0}$. For this reason a scalar multiple $\delta$ of $\Theta_{T}$ is called minimal if its inner part $\delta_{\text {inn }}$ coincides with $m_{T_{0}}$. Similarly, the $C_{.1}-C_{.0}$ decomposition (4) of $T$ is in fact a $C_{11}-C_{0}$ decomposition and $T_{1}, T_{0}^{\prime} \in(\mathrm{SM})$. Moreover, the invariant subspaces $\mathcal{H}_{0}$ and $\mathcal{H}_{1}$ defined by (3) and (4) satisfy $\mathcal{H}_{0} \cap \mathcal{H}_{1}=\{0\}$ and $\operatorname{span}\left(\mathcal{H}_{0}, \mathcal{H}_{1}\right)=\mathcal{H}$; and we have $\sigma(T)=\sigma\left(T_{0}\right) \cup \sigma\left(T_{1}^{\prime}\right)=\sigma\left(T_{1}\right) \cup \sigma\left(T_{0}^{\prime}\right)$ (see [SNF67, Section VIII.2.1]). Moreover, the spectrum of a $C_{11}$-contraction in (SM) is contained in $\mathbb{T}$ (see [SNF67, Proposition VI.4.3]).

Weak contractions. $T \in L(\mathcal{H})$ is called a weak contraction if $\mathbb{D} \backslash \sigma(T) \neq \emptyset$ and $I-T^{*} T \in S_{1}$. A weak contraction $T \in L(\mathcal{H})$ belongs to the class (SM) ([SNF67, Theorem VIII.1.1]). The contractions $T_{0}, T_{1}^{\prime}, T_{1}, T_{0}^{\prime}$ appearing in the $C_{0}-C_{11}$ and $C_{11}-C_{0}$ decompositions of $T$ are all weak contractions (see [SNF67, Theorem VIII.2.1]).

3. Smooth operators in $H^{\infty}(T)$. In this section we first find some necessary conditions for the spaces $H^{\infty}(T) \cap S_{\infty}$ not to be reduced to $\{0\}$. Then we explore in which cases these conditions are sufficient. All contrac- 
tions will be supposed to be c.n.u. Therefore we may work equivalently with $T$ or its canonical model $M_{\Theta}, \Theta=\Theta_{T}$, as $T$ and $M_{\Theta}$ are unitarily equivalent.

LEMma 3.1. Let $\Theta \in H^{\infty}\left(L\left(\mathcal{H}_{1}, \mathcal{H}_{2}\right)\right)$ be a contractive-valued function, and $P_{\Theta}$ defined as in Section 1. For $g \in L^{2}\left(\mathcal{H}_{1}\right)$, we have

$$
\left\|P_{\Theta}\left(\begin{array}{l}
0 \\
g
\end{array}\right)\right\|^{2}=\|g\|^{2}-\left\|P_{+} \Delta g\right\|^{2}=\|\Theta g\|^{2}+\left\|P_{-} \Delta g\right\|^{2},
$$

where $P_{+}$denotes the Riesz projection (i.e. onto the analytic part), and $P_{-}=I-P_{+}$.

Proof. The well known block decomposition of $P_{\Theta}$,

$$
P_{\Theta}=\left(\begin{array}{cc}
P_{+}-\Theta P_{+} \Theta^{*} & -\Theta P_{+} \Delta \\
-\Delta P_{+} \Theta^{*} & I-\Delta P_{+} \Delta
\end{array}\right),
$$

and the fact that $\Delta$ is a positive selfadjoint operator satisfying $\Delta^{2}=I-\Theta^{*} \Theta$ lead to

$$
\begin{aligned}
\left\|P_{\Theta}\left(\begin{array}{l}
0 \\
g
\end{array}\right)\right\|^{2} & =\left\|\Theta P_{+} \Delta g\right\|^{2}+\left\|\left(I-\Delta P_{+} \Delta\right) g\right\|^{2} \\
& =\left\|P_{+} \Delta g\right\|^{2}-\left\|\Delta P_{+} \Delta g\right\|^{2}+\left\|\left(I-\Delta P_{+} \Delta\right) g\right\|^{2} \\
& =\left\|P_{+} \Delta g\right\|^{2}-\left\|\Delta P_{+} \Delta g\right\|^{2}+\|g\|^{2}-2\left\|P_{+} \Delta g\right\|^{2}+\left\|\Delta P_{+} \Delta g\right\|^{2} \\
& =\|g\|^{2}-\left\|P_{+} \Delta g\right\|^{2},
\end{aligned}
$$

and $\|g\|^{2}-\left\|P_{+} \Delta g\right\|^{2}=\|\Theta g\|^{2}+\|\Delta g\|^{2}-\|\Delta g\|^{2}+\left\|P_{-} \Delta g\right\|^{2}=\|\Theta g\|^{2}+$ $\left\|P_{-} \Delta g\right\|^{2}$.

LEMma 3.2. Let $\Theta \in H^{\infty}\left(L\left(\mathcal{H}_{1}, \mathcal{H}_{2}\right)\right)$ be a contractive-valued function, and $M_{\Theta} \in L\left(K_{\Theta}\right)$ the model operator associated with $\Theta$. Assume that there exists $\varphi \in H^{\infty}, \varphi \neq 0$, such that $\varphi\left(M_{\Theta}\right)^{*} M_{\Theta}^{* n}$ tends SOT to 0 . Then $\Theta$ is an inner function, or equivalently, $M_{\Theta}^{* n}$ tends SOT to 0 .

Notice that both $M_{\Theta}^{n}$ and $M_{\Theta}^{* n}$ always tend WOT to 0 . Indeed, if $u, v \in$ $K_{\Theta} \subset L^{2}\left(\mathcal{D}_{T} \oplus \mathcal{D}_{T^{*}}\right)$, then

$$
\left\langle M_{\Theta}^{n} u, v\right\rangle=\left\langle P_{\Theta} z^{n} u, v\right\rangle=\left\langle z^{n} u, v\right\rangle=\int_{\mathbb{T}} \xi^{n}\langle u(\xi), v(\xi)\rangle d \mu(\xi)=\widehat{w}(-n),
$$

where $w=\langle u(\cdot), v(\cdot)\rangle \in L^{1}$; and therefore $\widehat{w}(-n) \rightarrow 0$ from the RiemannLebesgue lemma. Thus, for every c.n.u. contraction $T \in L(\mathcal{H}), T^{n}$ and $T^{* n}$ tend WOT to 0.

Proof of Lemma 3.2. Let $\varphi \in H^{\infty}$. First we compute the SOT limit of $\varphi\left(M_{\Theta}\right)^{*} M_{\Theta}^{* n}$ in the general case. Let $\left(\begin{array}{c}f \\ g\end{array}\right) \in K_{\Theta}$. Note that the function $z^{n} M_{\Theta}^{* n}\left(\begin{array}{c}f \\ g\end{array}\right)$ converges in $L^{2}\left(\mathcal{H}_{2} \oplus \mathcal{H}_{1}\right)$ to the function $\left(\begin{array}{l}0 \\ g\end{array}\right)$. This follows from 
the facts that

$$
M_{\Theta}^{* n}\left(\begin{array}{l}
f \\
g
\end{array}\right)=\left(\begin{array}{c}
P_{+} \bar{z}^{n} f \\
\bar{z}^{n} g
\end{array}\right),
$$

and that $\left\|P_{+} \bar{z}^{n} f\right\|=\sum_{k \geq n}\|\widehat{f}(k)\|^{2}$ tends to 0 in $L^{2}\left(\mathcal{H}_{2}\right)$. Then, for any $\varphi \in H^{\infty},\left\|\varphi\left(M_{\Theta}\right)^{*} M_{\Theta}^{* n}\left(\begin{array}{c}f \\ g\end{array}\right)\right\|^{2}$ converges to the same limit as

$$
\left\|P_{\Theta} \bar{\varphi}\left(\begin{array}{c}
0 \\
\bar{z}^{n} g
\end{array}\right)\right\|^{2}=\|\bar{\varphi} g\|^{2}-\left\|P_{+} \Delta \bar{z}^{n} \bar{\varphi} g\right\|^{2},
$$

where the last equality comes from Lemma 3.1. As $\Delta \bar{\varphi} g \in L^{2}\left(\mathcal{H}_{1}\right)$, the term $\left\|P_{+} \Delta \bar{z}^{n} \bar{\varphi} g\right\|^{2}=\left\|P_{+} \bar{z}^{n} \Delta \bar{\varphi} g\right\|^{2}$ tends to 0 . Therefore $\left\|\varphi\left(M_{\Theta}\right)^{*} M_{\Theta}^{* n}\left(\begin{array}{c}f \\ g\end{array}\right)\right\|$ tends to $\|\bar{\varphi} g\|$.

Now suppose that $\varphi$ is such that $\varphi\left(M_{\Theta}\right)^{*} M_{\Theta}^{* n}$ tends SOT to 0 . Then, for all $\left(\begin{array}{c}f \\ g\end{array}\right) \in K_{\Theta}$, we have $\|\bar{\varphi} g\|=0$, which implies that $g=0$. It remains to show that this result forces $\Delta$ to be identically 0 . Indeed, we then have

$$
K_{\Theta} \subset\left(\begin{array}{c}
H^{2}\left(\mathcal{H}_{2}\right) \\
0
\end{array}\right)
$$

and therefore

$$
K_{\Theta}^{\perp} \supset\left(\begin{array}{c}
0 \\
\operatorname{clos} \Delta L^{2}\left(\mathcal{H}_{1}\right)
\end{array}\right) .
$$

As $K_{\Theta}^{\perp}=\left(\begin{array}{c}\Theta \\ \Delta\end{array}\right) H^{2}\left(\mathcal{H}_{1}\right)$ contains no non-zero $\bar{z}$-invariant subspace, we necessarily have $\Delta=0$.

ThEOREM 3.3. Let $T \in L(\mathcal{H})$ be a c.n.u. contraction. If $H^{\infty}(T) \cap S_{\infty}$ $\neq\{0\}$, then $\Theta_{T} \in H^{\infty}\left(L\left(\mathcal{D}_{T}, \mathcal{D}_{T^{*}}\right)\right)$ is a two-sided inner function, in particular $\mathcal{D}_{T}$ and $\mathcal{D}_{T^{*}}$ are of the same dimension.

Proof. It suffices to show that $H^{\infty}(T) \cap S_{\infty} \neq\{0\}$ implies that $\Theta_{T}$ is inner. Then we can apply the result to $T^{*}$ and use the fact that $\Theta_{T^{*}}=\Theta_{T}^{\mathrm{t}}$. Let $M_{\Theta} \in L\left(K_{\Theta}\right)$ be the model of $T$. As $M_{\Theta}$ is unitarily equivalent to $T$, $H^{\infty}(T) \cap S_{\infty} \neq\{0\}$ if and only if $H^{\infty}\left(M_{\Theta}\right) \cap S_{\infty} \neq\{0\}$. Now, let $\varphi\left(M_{\Theta}\right) \in$ $S_{\infty}, \varphi \neq 0$. As $M_{\Theta}^{* n}$ tends WOT to $0, \varphi\left(M_{\Theta}\right)^{*} M_{\Theta}^{* n}$ tends SOT to 0 . From Lemma 3.2 we deduce that $\Theta$ is an inner function.

We are now interested in a converse to Theorem 3.3. Some additional assumptions are necessary to prevent the spectrum from behaving badly. The following lemmas concerning $C_{0}$-contractions will be useful.

LEMmA 3.4. Let $T \in L(\mathcal{H})$ be a $C_{0}$-contraction such that $\sigma(T) \cap \mathbb{D}$ is non-empty. If $\lambda \in \sigma(T) \cap \mathbb{D}$, then the Riesz projection $\mathcal{P}_{\lambda}$ belongs to $H^{\infty}(T)$.

Proof. For an outline of proof, see [SNF67, Section III.7.1].

Now we consider $C_{0}$-contractions $T$ with compact defect $D_{T}$ (equivalently $\left.I-T^{*} T \in S_{\infty}\right)$. Such contractions are said to be essentially uni- 
tary with respect to Fredholm theory (because $T \in C_{0}$, or more generally $\mathbb{D} \backslash \sigma(T) \neq \emptyset$, and $I-T^{*} T \in S_{\infty}$ imply that $T=U+K$, where $U$ is unitary and $K$ is compact).

Notice that for any c.n.u. contraction $T$ such that $\mathbb{D} \backslash \sigma(T) \neq \emptyset$ and $I-T^{*} T \in S_{\infty}, \sigma(T) \cap \mathbb{D}$ coincides with $\sigma_{\mathrm{p}}(T)$ and consists of an at most countable sequence of normal (finite multiplicity) eigenvalues tending to $\mathbb{T}$. In particular, the set of so-called normal eigenvalues, $\sigma_{\mathrm{np}}(T)$, coincides with $\sigma_{\mathrm{p}}(T)$ and $\sigma(T) \cap \mathbb{D}$.

REMARK 3.5. If $T \in L(\mathcal{H})$ is a c.n.u. contraction such that $H^{\infty}(T) \cap \mathcal{F}$ $\neq\{0\}$ then $T \in C_{0}$. Indeed, if $\varphi(T) \in \mathcal{F}$ with $\varphi \in H^{\infty}, \varphi \neq 0$, then the restriction of $T$ to $\varphi(T) \mathcal{H}$ has a minimal annihilating function, say $m$, and $m \varphi$ is a non-zero $H^{\infty}$ function annihilating $T$.

Now, we will see why, for an essentially unitary $C_{0}$-contraction $T$, we have $H^{\infty}(T) \cap S_{\infty} \neq\{0\}$. We start with a lemma which will have larger consequences. The method employed for the proof, which consists in using an outer function in $\mathcal{C}_{A}$ ( $H^{\infty}$ functions continuous on $\left.\overline{\mathbb{D}}\right)$ equal to zero, on a given set of Lebesgue measure zero, was introduced by B. Moore and E. Nordgren to study the existence of compact operators in the weakly closed algebra generated by an essentially unitary $C_{0}$-operator [MN75]. Another ingredient of this lemma consists in the characterization of compact Hankel operators in terms of their symbol; namely, for $\Phi \in L^{\infty}\left(L\left(\mathcal{H}_{1}, \mathcal{H}_{2}\right)\right)$, the Hankel operator $H_{\Phi}$ is compact if and only if $\Phi \in H^{\infty}\left(L\left(\mathcal{H}_{1}, \mathcal{H}_{2}\right)\right)+$ $\mathcal{C}\left(\mathbb{T}, S_{\infty}\left(\mathcal{H}_{1}, \mathcal{H}_{2}\right)\right)$, where $\mathcal{C}(\mathbb{T}, X)$ stands for the space of $X$-valued continuous functions on $\mathbb{T}$ (see [Muh69], [Muh71], [BP75]). Recall that $H_{\Phi}$ is defined by

$$
H_{\Phi}: H^{2}\left(\mathcal{H}_{1}\right) \rightarrow H_{-}^{2}\left(\mathcal{H}_{2}\right)=L^{2}\left(\mathcal{H}_{2}\right) \ominus H^{2}\left(\mathcal{H}_{2}\right), \quad h \mapsto P_{-} \Phi h .
$$

Lemma 3.6. Let $T \in L(\mathcal{H})$ be a $C_{0}$-contraction such that $I-T^{*} T \in S_{\infty}$, and $m_{T}$ the minimal function of $T$. Then there exists $\varphi \in H^{\infty}$ such that $\varphi(T) \neq 0$ and the Hankel operator $H_{\varphi \Theta_{T}^{*}}$ is compact. More precisely, we can take $\varphi=f m_{T} / m_{1}$, where $m_{1}$ is any non-trivial inner factor of $m_{T}$ such that $\sigma\left(m_{1}\right) \cap \mathbb{T}$ is of Lebesgue measure zero, and $f$ is any outer $\mathcal{C}_{A}$ function equal to zero on $\sigma\left(m_{1}\right) \cap \mathbb{T}$.

Proof. Take a non-trivial factorization $m_{T}=m_{1} m_{2}$ such that $m_{1}, m_{2}$ are inner and $\sigma\left(m_{1}\right) \cap \mathbb{T}$ is contained in a closed subset $\gamma$ of $\mathbb{T}$ of Lebesgue measure $\mu(\gamma)=0$. From a theorem of Fatou we obtain the existence of an outer function $f \in \mathcal{C}_{A}$ such that $\left.f\right|_{\gamma}=0$. Set $\varphi=f m_{2}$. Then $\varphi / m_{T}=$ $f / m_{1} \notin H^{\infty}$, and the minimality of $m_{T}$ implies that $\varphi(T) \neq 0$. Now, there exists an invariant subspace $E \subset \mathcal{H}$ of $T$ such that $m_{1}$ and $m_{2}$ annihilate $T_{1}=\left.T\right|_{E} \in L(E)$ and $T_{2}=\left.P_{E^{\perp}} T\right|_{E^{\perp}} \in L\left(E^{\perp}\right)$ respectively, where $P_{E^{\perp}}$ denotes the orthogonal projection from $\mathcal{H}$ onto $E^{\perp}$ (see [SNF67, Theorem 
III.6.3]). Moreover, there exist $\Theta_{1} \in H^{\infty}\left(L\left(\mathcal{D}_{T}, \mathcal{K}\right)\right), \Theta_{2} \in H^{\infty}\left(L\left(\mathcal{K}, \mathcal{D}_{T^{*}}\right)\right)$, where $\mathcal{K}$ is an auxiliary separable Hilbert space, such that $\Theta=\Theta_{2} \Theta_{1}$ and $\Theta_{T_{1}}$ and $\Theta_{T_{2}}$ coincide with the pure part of $\Theta_{1}$ and $\Theta_{2}$ respectively (see [SNF67, Theorem VII.1.1 and Proposition VII.2.1]). Then, for $i=1,2$, the function $m_{i}$ is a scalar multiple of $\Theta_{T_{i}}$ (see [Ber88, Proposition V.3.2 and Corollary V.3.3]) and $\Theta_{T_{i}}$ is a two-sided inner function. The same properties hold for $\Theta_{i}$ as $\Theta_{i}=\Theta_{T_{i}} \oplus U_{i}$, where $U_{i}$ is a unitary operator. Let $\Omega_{1} \in$ $H^{\infty}\left(L\left(\mathcal{K}, \mathcal{D}_{T}\right)\right)$ and $\Omega_{2} \in H^{\infty}\left(L\left(\mathcal{D}_{T^{*}}, \mathcal{K}\right)\right)$ be such that $\Theta_{1} \Omega_{1}=m_{1} I$ and $\Theta_{2} \Omega_{2}=m_{2} I$. Now,

$$
\varphi \Theta^{*}=f m_{2} \Theta_{1}^{*} \Theta_{2}^{*}=f \Theta_{1}^{*}\left(m_{2} \Theta_{2}^{*}\right)=f \Theta_{1}^{*} \Omega_{2} .
$$

The proof will be completed by showing that $f \Theta_{1}^{*} \in H^{\infty}\left(L\left(\mathcal{K}, \mathcal{D}_{T}\right)\right)+$ $\mathcal{C}\left(\mathbb{T}, S_{\infty}\left(\mathcal{K}, \mathcal{D}_{T}\right)\right)$, as this implies that $H_{f \Theta_{1}^{*}}$ is compact, and then so is $H_{f \Theta_{1}^{*} \Omega_{2}}=H_{\varphi \Theta^{*}}$. Compactness of $I-T^{*} T$ implies that $\left.P_{E}\left(I-T^{*} T\right)\right|_{E}$ $=I-T_{1}^{*} T_{1}$ is compact; and thus $\Theta_{1}(z)=A+K(z), z \in \mathbb{D}$, where $A \in$ $L\left(\mathcal{D}_{T}, \mathcal{K}\right)$ and $K \in H^{\infty}\left(S_{\infty}\left(\mathcal{D}_{T}, \mathcal{K}\right)\right)$. Therefore it suffices to show that $f \Theta_{1}^{*}$ considered as a function on $\mathbb{T}$ is continuous at every point of $\mathbb{T}$, as this fact implies that $K \in \mathcal{C}\left(S_{\infty}\left(\mathcal{D}_{T}, \mathcal{K}\right)\right)$. As $\sigma\left(\Theta_{T_{1}}\right)=\sigma\left(T_{1}\right)=\sigma\left(m_{1}\right) \subset \gamma$, the function $\Theta_{T_{1}}$ has a holomorphic continuation at every $\xi \in \mathbb{T} \backslash \gamma$, and so does $\Theta_{1}$. It follows that $f \Theta_{1}^{*}$ is continuous at every $\xi \in \mathbb{T} \backslash \gamma$. Eventually, if $\xi \in \gamma$, then $f(\xi)=0$. As $\Theta_{1}^{*}$ is bounded on $\mathbb{T}$, the continuity of $f$ implies that of $f \Theta_{1}^{*}$ at $\xi$.

Before giving a direct consequence of Lemma 3.6 we deduce the following fact which will be useful later on (see Subsection 4.2).

Lemma 3.7. Let $T \in(\mathrm{SM}), T \notin C_{11}$, be such that $I-T^{*} T \in S_{\infty}$. Then there exists $\psi \in H^{\infty}$ such that $H_{\psi \Theta_{T}^{-1}}$ is compact but

$$
\psi \Theta_{T}^{-1} \notin H^{\infty}\left(L\left(\mathcal{D}_{T^{*}}, \mathcal{D}_{T}\right)\right) .
$$

More precisely, we can take $\psi=f \delta / m_{1}$, where $\delta$ is a minimal scalar multiple of $T, m_{1}$ is any non-trivial inner factor of $\delta$ such that $\sigma\left(m_{1}\right) \cap \mathbb{T}$ is of Lebesgue measure zero, and $f$ is any outer function in $\mathcal{C}_{A}$ equal to zero on $\sigma\left(m_{1}\right) \cap \mathbb{T}$.

Proof. Denote by $T_{0}$ the $C_{0}$-part of $T$ appearing in the $C_{0}-C_{11}$ decomposition of $T$. This $T_{0}$ is the restriction of $T$ to $\mathcal{H}_{0} \neq\{0\}$ as $T \notin C_{11}$. Therefore $\sigma\left(T_{0}\right) \subset \sigma(T)$ and $I-T_{0}^{*} T_{0}=\left.P_{\mathcal{H}_{0}}\left(I-T^{*} T\right)\right|_{\mathcal{H}_{0}}$ is compact. On the other hand, the characteristic function of $T_{0}$ is the pure part of $\Theta_{\text {inn }}$ (see [SNF67, Theorem VII.1.1 and Proposition VII.2.1]). If there exists $h \in H^{\infty} \backslash\{0\}$ such that $h(T)=0$, then $T$ is in fact $C_{0}$ (i.e. $T$ coincides with $T_{0}$ ), and the result is given by Lemma 3.6. Thus we can suppose that $h(T) \neq 0$ for every $h \in H^{\infty} \backslash\{0\}$. Lemma 3.6 applied to $T_{0}$ gives $\varphi=f m_{T_{0}} / m_{1}$ such that $H_{\varphi \Theta_{T_{0}}^{*}}$ is compact. Let $\Theta_{T}=\Theta_{\text {out }} \Theta_{\text {inn }}$ be the outer-inner factorization 
of $\Theta_{T}$. In fact $\Theta_{T_{0}}$ coincides with the pure part of $\Theta_{\text {inn }}$ (see [SNF67, Theorem VII.1.1 and Proposition VII.2.1]); thus $H_{\varphi \Theta_{i n n}^{*}}$ is also compact. Let $\delta$ be a minimal scalar multiple of $\Theta_{T}$, that is, $\delta=m_{T_{0}} \delta_{\text {out }}$ with $\delta_{\text {out }}$ outer. Set

$$
\psi=\delta_{\text {out }} \varphi=f \frac{m_{T_{0}}}{m_{1}} \delta_{\text {out }}=f \frac{\delta}{m_{1}} .
$$

Then $\psi \Theta_{T}^{-1}=\varphi \Theta_{\text {inn }}^{*} \delta_{\text {out }} \Theta_{\text {out }}^{-1}$; thus $H_{\psi \Theta_{T}^{-1}}$ is compact. Further, if we suppose that $\psi \Theta_{T}^{-1}=F \in H^{\infty}\left(L\left(\mathcal{D}_{T^{*}}, \mathcal{D}_{T}\right)\right)$, then $\psi I=\Theta_{T} T=F \Theta_{T}$. Therefore, $\psi=\delta_{\text {out }} m_{T_{0}} / m_{1}$ is a scalar multiple of $\Theta_{T}$, which is impossible since $\delta_{\text {out }} m_{T_{0}}$ is a minimal scalar multiple.

The first part of the following theorem is mostly known [Nor75] but we give a proof for the sake of completeness.

Theorem 3.8. Let $T \in L(\mathcal{H})$ be a $C_{0}$-contraction such that $I-T^{*} T$ $\in S_{\infty}$. Then $H^{\infty}(T) \cap S_{\infty} \neq\{0\}$. Moreover, $H^{\infty}(T) \cap \mathcal{F} \neq\{0\}$ if and only if $\sigma(T) \cap \mathbb{D} \neq \emptyset$.

Proof. To prove the first assertion we use Lemma 3.6 and get a function $\varphi \in H^{\infty}$ such that $\varphi(T) \neq 0$ and $H_{\varphi \Theta_{T}^{*}}$ is compact. As $T$ is $C_{0}$, its characteristic function $\Theta$ is two-sided inner. Then $P_{\Theta}=\Theta P_{-} \Theta^{*}$ and, identifying $T$ with its model operator $M_{\Theta}$, we deduce that $\varphi(T)=\left.\Theta H_{\varphi \Theta_{T}^{*}}\right|_{K_{\Theta}}$ is compact. Now prove the second assertion. If $H^{\infty}(T) \cap \mathcal{F} \neq\{0\}$, then $T$ has a non-trivial finite-dimensional invariant subspace, and therefore an eigenvalue. The converse is an immediate consequence of Lemma 3.4. Indeed, if $\lambda \in \sigma(T) \cap \mathbb{D}$, then $\mathcal{P}_{\lambda} \in H^{\infty}(T) \cap \mathcal{F}$.

Yet another proof is to observe directly that

$$
m_{T}(T)=b_{\lambda}(T) \frac{m_{T}}{b_{\lambda}}(T)=0,
$$

whence

$$
\frac{m_{T}}{b_{\lambda}}(T) \subset \operatorname{ker} b_{\lambda}(T),
$$

and $\operatorname{ker} b_{\lambda}(T)$ is finite-dimensional as $\lambda$ is necessarily of finite type. Here $b_{\lambda}$ denotes the elementary Blaschke factor,

$$
b_{\lambda}(z)=\frac{|\lambda|}{\lambda} \frac{\lambda-z}{1-\bar{\lambda} z}
$$

Corollary 3.9. Let $T \in L(\mathcal{H})$ be an (SM)-contraction such that $I-$ $T^{*} T \in S_{\infty}$. Then $H^{\infty}(T) \cap S_{\infty} \neq\{0\}$ if and only if $\Theta_{T}$ is a two-sided inner function.

Proof. If $\Theta_{T}$ is inner, then $T \in C_{0}$ and the result follows from Theorem 3.8. The converse is a consequence of Theorem 3.3.

Now, we consider $C_{00}$-contractions $T$ (that is, contractions having a twosided inner characteristic function) subject to restrictions of "smoothness" of 
the defect operator $D_{T}=\left(I-T^{*} T\right)^{1 / 2}$. The following result means that only the trace class smoothness of $D_{T}^{2}$ can guarantee the existence of compact $H^{\infty}$ functions of $T$.

THEOREM 3.10. Let $\mathfrak{S} \subset S_{\infty}$ be a symmetrically normed ideal of $L(\mathcal{H})$. The following are equivalent:

(i) For every c.n.u. contraction $T \in C_{00}$ such that $\mathbb{D} \backslash \sigma(T) \neq \emptyset$ and $I-T^{*} T \in \mathfrak{S}$, we have

(ii) $\mathfrak{S}=S_{1}$.

$$
H^{\infty}(T) \cap S_{\infty} \neq\{0\}
$$

We refer to [GK69] for properties of symmetrically normed ideals of $L(\mathcal{H})$. For the proof of Theorem 3.10 we need the following notion. Let $\sigma \subset \mathbb{D}$. Then $\sigma$ is called a determining subset for (the $H^{\infty}$ norm on) $\mathbb{D}$ if $\|f\|_{\infty}=\sup _{z \in \sigma}|f(z)|$ for all $f \in H^{\infty}=H^{\infty}(\mathbb{D})$. The following lemma is a result by N. K. Nikolski and S. A. Vinogradov (see [Nik71]).

Lemma 3.11. Suppose $0<r_{n}<1(n \geq 1)$ satisfy $\lim r_{n}=1$. There exists a determining sequence for $\mathbb{D}$, say $\left(\lambda_{n}\right)_{n \geq 1}$, such that $\left|\lambda_{n}\right|=r_{n}(n \geq 1)$ if and only if $\sum_{n \geq 1}\left(1-r_{n}\right)=\infty$.

Proof of Theorem 3.10. The fact that property (i) is true when $\mathfrak{S}=S_{1}$ can be deduced from Theorem 3.8. Indeed, in this case $T$ is a weak contraction in $C_{00}$, and therefore $T \in C_{0}$. To prove that (i) implies (ii) suppose that $\mathfrak{S} \neq S_{1}$. Take $A \in \mathfrak{S} \backslash S_{1}$ and let $A=\sum_{n>0} s_{n}\left\langle\cdot, x_{n}\right\rangle y_{n}$ be a Schmidt decomposition of $A$, that is, $\left(s_{n}\right)_{n \geq 0}$ is a sequence of positive numbers decreasing to 0 , and $\left(x_{n}\right)_{n \geq 0}$ and $\left(y_{n}\right)_{n \geq 0}$ are orthonormal families in $\mathcal{H}$. As $A \notin S_{1}$, we have $\sum_{n \geq 0} s_{n}=\infty$, and hence $s_{n} \neq 0$ for all $n \geq 0$. With $\mathfrak{S}$ being an ideal, we can suppose that $\left(x_{n}\right)_{n \geq 0}$ and $\left(y_{n}\right)_{n \geq 0}$ coincide with the same orthonormal basis $\left(e_{n}\right)_{n \geq 0}$ of $\mathcal{H}$. Define $T=\operatorname{diag}\left(\lambda_{n}\right)_{n \geq 0}$ with respect to this basis, where we choose $\left(\lambda_{n}\right)_{n \geq 0} \subset \mathbb{D}$ to be a determining subset for $\mathbb{D}$ satisfying $\left|\lambda_{n}\right|^{2}=1-s_{n}$, which is possible due to Lemma 3.11, where we take $r_{n}=\sqrt{1-s_{n}}$. Under these conditions, $T$ is a c.n.u. $C_{00}$-contraction and $I-T^{*} T=\sum_{n \geq 0} s_{n}\left\langle\cdot, e_{n}\right\rangle e_{n} \in \mathfrak{S}$. But if $\varphi \in H^{\infty}$, then $\varphi(T)=\operatorname{diag}\left(\varphi\left(\lambda_{n}\right)\right)_{n \geq 0}$, thus $\varphi(T) \in S_{\infty}$ if and only if $\lim _{n \rightarrow \infty} \varphi\left(\lambda_{n}\right)=0$, which implies that $\varphi=0$. Indeed, if $\left(\lambda_{n}\right)_{n \geq 0} \subset \mathbb{D}$ is a determining sequence for $\mathbb{D}$, then so is $\left(\lambda_{n}\right)_{n \geq N} \subset \mathbb{D}$ for every $N \geq 0$. Therefore $\|\varphi\|_{\infty}=\sup _{n \geq N}\left|\varphi\left(\lambda_{n}\right)\right|$, which tends to 0 as $N \rightarrow \infty$.

\section{Smooth operators in the commutant}

\subsection{Finite-rank operators in the commutant}

TheOREM 4.1. Let $T \in L(\mathcal{H})$ be a c.n.u. contraction such that $\mathbb{D} \backslash$ $\sigma(T) \neq \emptyset$. Suppose $I-T^{*} T \in S_{\infty}$. Then $\{T\}^{\prime} \cap \mathcal{F} \neq\{0\}$ if and only if $\mathbb{D} \cap \sigma(T)$ is non-empty. The condition $\mathbb{D} \backslash \sigma(T) \neq \emptyset$ cannot be omitted. 
Proof. First suppose that $\{T\}^{\prime} \cap \mathcal{F} \neq\{0\}$. Let $A \in\{T\}^{\prime} \cap \mathcal{F}, A \neq 0$. Set $E=A \mathcal{H} \subset \mathcal{H}$. Then $E \neq\{0\}$ is a finite-dimensional invariant subspace of $T$. Therefore, $\left.T\right|_{E} \in L(E)$ admits an eigenvalue $\lambda$. Then $\lambda$ is also an eigenvalue of $T$ and has to be in $\mathbb{D}$.

Now suppose $\mathbb{D} \cap \sigma(T) \neq \emptyset$. Then $\sigma_{\mathrm{np}}(T)=\sigma_{\mathrm{p}}(T)=\mathbb{D} \cap \sigma(T) \neq \emptyset$, therefore there exists $\lambda \in \mathbb{D}$ which is a normal eigenvalue of $T$. Consequently, the corresponding Riesz projection $\mathcal{P}_{\lambda} \neq 0$ is of finite rank. As $\mathcal{P}_{\lambda}$ belongs to $\{T\}^{\prime}$, we deduce that $\{T\}^{\prime} \cap \mathcal{F}$ cannot be reduced to $\{0\}$.

Finally, consider the case when $T=S$, the shift operator on $H^{2}$. Then $I-S^{*} S=0 \in S_{\infty}$ but $\sigma(T) \supset \mathbb{D}$. Moreover $\{S\}^{\prime}=H^{\infty}(S)$ obviously contains no non-zero finite-rank operator, and even no non-zero compact operator.

4.2. Compact operators in the commutant. First, we note that the condition $\{T\}^{\prime} \cap S_{\infty} \neq\{0\}$ is not in general sufficient for $\Theta_{T}$ to be a two-sided inner function (in contrast to the case of $H^{\infty}(T)$, see Theorem 3.3). For example, it is easy to see that if $\Theta$ is a scalar $H^{\infty}$ function and $\Theta=0$, then $M_{\Theta}$ can be identified with $S \oplus S^{*}$, where $S$ is the shift operator on $H^{2}$, and

$$
\left(\begin{array}{cc}
0 & 0 \\
\Gamma_{\varphi} & 0
\end{array}\right) \in\left\{M_{\Theta}\right\}^{\prime} \cap S_{\infty} \quad \text { for every } \varphi \in H^{\infty}+\mathcal{C}(\mathbb{T})
$$

where $\Gamma_{\varphi}$ denotes the Hankel operator on $H^{2}$ with symbol $\varphi$. Thus we then have $\{T\}^{\prime} \cap S_{\infty} \neq\{0\}$.

Now, we give a characterization of operators $Y$ on $H^{2}\left(\mathcal{D}_{T^{*}}\right) \oplus \overline{\Delta L^{2}\left(\mathcal{D}_{T}\right)}$ given by formula (1) which are liftings of compact operators in $\left\{M_{\Theta}\right\}^{\prime}$.

Lemma 4.2. Let $T \in L(\mathcal{H})$ be a c.n.u. contraction and $\Theta=\Theta_{T}$. If $X \in\left\{M_{\Theta}\right\}^{\prime}$ is represented via the CLT by $X=\left.P_{\Theta} Y\right|_{K_{\Theta}}$, then $X$ is compact if and only if the operator $P_{\Theta} Y$ acting on

$$
\left(\begin{array}{c}
H^{2}\left(\mathcal{D}_{T^{*}}\right) \\
\operatorname{clos} \Delta L^{2}\left(\mathcal{D}_{T}\right)
\end{array}\right)
$$

is compact.

Proof. This is an immediate consequence of the condition

$$
Y\left(\begin{array}{c}
\Theta \\
\Delta
\end{array}\right) H^{2}\left(\mathcal{D}_{T}\right) \subset\left(\begin{array}{c}
\Theta \\
\Delta
\end{array}\right) H^{2}\left(\mathcal{D}_{T}\right)
$$

Lemma 4.3. Let $T \in L(\mathcal{H})$ be an (SM)-contraction and $\Theta=\Theta_{T}$. Let $X \in\left\{M_{\Theta}\right\}^{\prime}$ be represented via the CLT by $X=\left.P_{\Theta} Y\right|_{K_{\Theta}}$, where $Y$ is the lifting of $X$ with parameters $A_{1}, B_{1}, C_{1}$ given by formula (1). If $X$ is compact, then $C_{1}=0$. 
Proof. If $X$ is compact, it follows from Lemma 4.2 that $P_{\Theta} Y$ is compact. Then $\left.P_{\Theta} Y\right|_{0 \oplus \overline{\Delta L^{2}\left(\mathcal{D}_{T}\right)}}$ is also compact. If $g \in \overline{\Delta L^{2}\left(\mathcal{D}_{T}\right)}$, then

$$
Y\left(\begin{array}{l}
0 \\
g
\end{array}\right)=\left(\begin{array}{c}
0 \\
C_{1} g
\end{array}\right)
$$

According to Lemma 3.1 we have

$$
\left\|P_{\Theta} Y\left(\begin{array}{l}
0 \\
g
\end{array}\right)\right\|^{2}=\left\|\Theta C_{1} g\right\|^{2}+\left\|P_{-} \Delta C_{1} g\right\|^{2} \geq\left\|\Theta C_{1} g\right\|^{2} .
$$

Therefore the map $g \in \overline{\Delta L^{2}\left(\mathcal{D}_{T}\right)} \mapsto \Theta C_{1} g$ is compact. Let $\delta \in H^{\infty}$ be a scalar multiple of $\Theta$, and $\Omega \in H^{\infty}\left(L\left(\mathcal{D}_{T^{*}}, \mathcal{D}_{T}\right)\right)$ be such that $\Omega \Theta=\delta$. Then $g \mapsto \Omega \Theta C_{1} g=\delta C_{1} g$ is compact, which implies that $C_{1}(\xi) \Delta(\xi)=0$ for a.e. $\xi \in \mathbb{T}$. Indeed, if there exists $g_{0}$ such that $\left\|\delta(\xi) C_{1}(\xi) g_{0}(\xi)\right\| \geq \varepsilon>0$ on a set of positive measure then the operator $h \mapsto \delta C_{1} g_{0} h$ cannot be compact. Therefore $C_{1}=0$ as $C_{1} \in L^{\infty}\left(L\left(\overline{\Delta L^{2}\left(\mathcal{D}_{T}\right)}\right)\right)$.

Lemma 4.4. Let $T \in L(\mathcal{H})$ be an $(\mathrm{SM})$-contraction and $\Theta=\Theta_{T}$. Let $X \in\left\{M_{\Theta}\right\}^{\prime}$ be represented via the CLT by $X=\left.P_{\Theta} Y\right|_{K_{\Theta}}$, where $Y$ is the lifting of $X$ with parameters $A_{1}, B_{1}, C_{1}$ given by (1). If $C_{1}=0$ then

$$
P_{\Theta} Y=\left(\begin{array}{c}
\Theta \\
\Delta
\end{array}\right) P_{-} \Theta^{-1} A_{*} P_{*},
$$

where $P_{*}$ stands for the orthogonal projection from $H^{2}\left(\mathcal{D}_{T^{*}}\right) \oplus \overline{\Delta L^{2}\left(\mathcal{D}_{T}\right)}$ onto $H^{2}\left(\mathcal{D}_{T^{*}}\right)$ and $A_{*} \in H^{\infty}\left(L\left(\mathcal{D}_{T^{*}}\right)\right)$ is such that $\Theta^{-1} A_{*} \Theta \in H^{\infty}\left(L\left(\mathcal{D}_{T}\right)\right)$. Conversely, if $P_{\Theta} Y$ satisfies (6), with $A_{*} \in H^{\infty}\left(L\left(\mathcal{D}_{T^{*}}\right)\right)$ such that $\Theta^{-1} A_{*} \Theta$ $\in H^{\infty}\left(L\left(\mathcal{D}_{T}\right)\right)$, then $X=\left.P_{\Theta} Y\right|_{K_{\Theta}} \in\left\{M_{\Theta}\right\}^{\prime}$.

Proof. Suppose $X \in\left\{M_{\Theta}\right\}^{\prime} \cap S_{\infty}$ and $Y$ is a lifting of $X$ with parameters $A, A_{*}$ and $B$ given by (2). The fact that $C_{1}=0$ in (1) means that $\Delta A \Delta=B \Theta$. As $T \in(\mathrm{SM}), \Theta$ is invertible a.e. on $\mathbb{T}$ and we can write $B=\Delta A \Delta \Theta^{-1}$. Then we use the following two intertwining relations: $\Theta A=A_{*} \Theta$ and $\Theta \Delta=\Delta_{*} \Theta$. We deduce that $B=\Delta A \Theta^{-1} \Delta_{*}=\Delta \Theta^{-1} A_{*} \Delta_{*}$ and $B \Delta_{*}=\Delta A \Theta^{-1}\left(1-\Theta \Theta^{*}\right)=\Delta A\left(\Theta^{-1}-\Theta^{*}\right)$. Therefore $\Delta A \Theta^{*}+B \Delta_{*}=$ $\Delta A \Theta^{-1}=\Delta \Theta^{-1} A_{*}$ and $Y$ has the following form:

$$
Y=\left(\begin{array}{cc}
A_{*} & 0 \\
\Delta \Theta^{-1} A_{*} & 0
\end{array}\right) .
$$

We compute $P_{\Theta} Y$ using the block matrix decomposition (5) and the following identities:

$$
\begin{aligned}
1-\Theta P_{+} \Theta^{*}-\Theta P_{+} \Delta^{2} \Theta^{-1} & =\Theta\left(1-P_{+} \Theta^{*} \Theta-P_{+} \Delta^{2}\right) \Theta^{-1} \\
& =\Theta\left(1-P_{+}\right) \Theta^{-1}=\Theta P_{-} \Theta^{-1} \\
-\Delta P_{+} \Theta^{*}+\left(1-\Delta P_{+} \Delta\right) \Delta \Theta^{-1} & =\Delta\left(-P_{+} \Theta^{*} \Theta+1-P_{+} \Delta^{2}\right) \Theta^{-1} \\
& =\Delta\left(1-P_{+}\right) \Theta^{-1}
\end{aligned}
$$


The result follows. The converse is clear because $Y$, as defined by (7), with $A_{*} \in H^{\infty}\left(L\left(\mathcal{D}_{T^{*}}\right)\right)$ such that $\Theta^{-1} A_{*} \Theta \in H^{\infty}\left(L\left(\mathcal{D}_{T^{*}}\right)\right)$, satisfies the required conditions for $Y$ to be a lifting.

Corollary 4.5. Let $T \in L(\mathcal{H})$ be an $(\mathrm{SM})$-contraction and $\Theta=\Theta_{T}$. Let $X \in\left\{M_{\Theta}\right\}^{\prime}$. Then $X$ is compact if and only if

$$
X=\left.\left(\begin{array}{c}
\Theta \\
\Delta
\end{array}\right) P_{-} \Theta^{-1} A_{*} P_{*}\right|_{K_{\Theta}}
$$

for some $A_{*} \in H^{\infty}\left(L\left(\mathcal{D}_{T^{*}}\right)\right)$ satisfying $\Theta^{-1} A_{*} \Theta \in H^{\infty}\left(L\left(\mathcal{D}_{T^{*}}\right)\right)$ and $\Theta^{-1} A_{*}$ $\in H^{\infty}\left(L\left(\mathcal{D}_{T^{*}}, \mathcal{D}_{T}\right)\right)+\mathcal{C}\left(\mathbb{T}, S_{\infty}\left(\mathcal{D}_{T^{*}}, \mathcal{D}_{T}\right)\right)$, where $P_{*}$ stands for the orthogonal projection from $H^{2}\left(\mathcal{D}_{T^{*}}\right) \oplus \overline{\Delta L^{2}\left(\mathcal{D}_{T}\right)}$ onto $H^{2}\left(\mathcal{D}_{T^{*}}\right)$. In this case, $X \neq 0$ if and only if $\Theta^{-1} A_{*} \notin H^{\infty}\left(L\left(\mathcal{D}_{T^{*}}, \mathcal{D}_{T}\right)\right)$.

Proof. If $X$ is compact we get the expression of $X$ by Lemmas 4.2 and 4.4. As the operator $\left(\begin{array}{l}\Theta \\ \Delta\end{array}\right)$ is an isometry the compactness of such an $X$ is equivalent to the compactness of $H_{\Theta^{-1}} A_{*}$ and also to $\Theta^{-1} A_{*}$ belonging to $H^{\infty}\left(L\left(\mathcal{D}_{T^{*}}, \mathcal{D}_{T}\right)\right)+\mathcal{C}\left(\mathbb{T}, S_{\infty}\left(\mathcal{D}_{T^{*}}, \mathcal{D}_{T}\right)\right)$. Moreover, $X=0$ is equivalent to $P_{-} \Theta^{-1} A_{*}=0$. Conversely, if $X$ is defined by (8) the last assertion of Lemma 4.4 tells us that $X$ is in $\left\{M_{\Theta}\right\}^{\prime}$.

Remark 4.6. The conclusions of Lemmas 4.3 and 4.4 and of Corollary 4.5 remain valid if we replace the condition $T \in(\mathrm{SM})$ by the weaker condition that $\Theta_{T}(\xi)$ be invertible a.e. on $\mathbb{T}$.

For $T \in(\mathrm{SM})$, the following theorem gives a necessary and sufficient condition for $\{T\}^{\prime} \cap S_{\infty} \neq\{0\}$ in terms of $\Theta_{T}$, namely $\Theta_{T}$ must be neither outer nor $*$-outer.

TheOrem 4.7. Let $T \in L(\mathcal{H})$ be a c.n.u. contraction. If $T \in C_{1} \cup C_{.1}$ (equivalently $\Theta_{T}$ is either outer or *-outer) then $\{T\}^{\prime} \cap S_{\infty}=\{0\}$. If moreover $T \in(\mathrm{SM})$ and $I-T^{*} T \in S_{\infty}$, then the converse is also true, and in fact, $\{T\}^{\prime} \cap S_{\infty}=\{0\}$ implies that $T \in C_{11}$.

Proof. To prove the first assertion suppose that there exists a non-zero $K \in\{T\}^{\prime} \cap S_{\infty}$. As $T^{n}$ tends WOT to 0 , we deduce that $K T^{n}$ tends SOT to 0 . But $K T^{n}=T^{n} K$ for all $n \geq 0$. Therefore $\left.T^{n}\right|_{\operatorname{Im} K}$ tends SOT to 0 . As $\operatorname{Im} K \neq\{0\}, T \notin C_{1}$. The same reasoning applies to $T^{*}$, thus $T^{*} \notin C_{1}$, or equivalently $T \in C_{.1}$.

Now suppose that $T \in(\mathrm{SM}), I-T^{*} T \in S_{\infty}$ and $T \notin C_{11}$. As $T \in(\mathrm{SM})$, that means $T \notin C_{1} . \cup C_{.1}$. Therefore $\Theta=\Theta_{T}$ is not outer. By Corollary 4.5, it remains to find $A_{*} \in H^{\infty}\left(L\left(\mathcal{D}_{T^{*}}\right)\right)$ such that $\Theta^{-1} A_{*} \Theta \in H^{\infty}\left(L\left(\mathcal{D}_{T}\right)\right)$ and $\Theta^{-1} A_{*} \in H^{\infty}\left(L\left(\mathcal{D}_{T^{*}}, \mathcal{D}_{T}\right)\right)+\mathcal{C}\left(\mathbb{T}, S_{\infty}\left(\mathcal{D}_{T^{*}}, \mathcal{D}_{T}\right)\right)$, but $P_{-} \Theta^{-1} A_{*} \neq 0$. But we know from Lemma 3.7 that there exists $u \in H^{\infty}$ such that $u \Theta^{-1} \in$ $H^{\infty}\left(L\left(\mathcal{D}_{T^{*}}, \mathcal{D}_{T}\right)\right)+\mathcal{C}\left(S_{\infty}\left(\mathcal{D}_{T^{*}}, \mathcal{D}_{T}\right)\right)$ with $u \Theta^{-1} \notin H^{\infty}\left(L\left(\mathcal{D}_{T^{*}}, \mathcal{D}_{T}\right)\right)$. The choice of $A_{*}=u I$ gives the result. 
4.3. Finite-rank approximation of compact operators in the commutant. In this subsection we are interested in the density of finite-rank operators in the commutant of a given c.n.u. contraction $T \in L(\mathcal{H})$ in the space $\{T\}^{\prime} \cap S_{\infty}$ of compact operators from the commutant.

If $B \in L(\mathcal{H})$ and $\lambda \in \sigma_{\mathrm{p}}(B)$, we denote by $C_{\lambda}(B)=\bigcup_{N \geq 1} \operatorname{ker}(B-\lambda)^{N}$ the root manifold corresponding to the eigenvalue $\lambda$ of $B$. We denote by

$$
C(B)=\bigcup_{\lambda \in \sigma_{\mathrm{p}}(B)} C_{\lambda}(B)
$$

the set consisting of all generalized eigenvectors (root vectors) of $B$. Now $B$ is said to be complete if the family of generalized eigenvectors of $B$ is total in $X$, so that $X=\operatorname{clos} C(B)$.

Let $T \in L(\mathcal{H})$ be a c.n.u. contraction, and define $E_{T}$ to be the total image of $\{T\}^{\prime} \cap S_{\infty}$, that is,

$$
E_{T}=\operatorname{clos}\left(\bigcup\left\{X \mathcal{H}: X \in\{T\}^{\prime} \cap S_{\infty}\right\}\right) .
$$

Then $E_{T}$ is hyperinvariant for $T$, that is, invariant for every $X \in\{T\}^{\prime}$. We set $T_{E}=\left.T\right|_{E_{T}}$.

LEMma 4.8. In the notation above, if $\{T\}^{\prime} \cap \mathcal{F}$ is SOT dense in $\{T\}^{\prime} \cap$ $S_{\infty}$ then $T_{E}$ is complete.

Proof. Let $X \in\{T\}^{\prime} \cap S_{\infty}$ and $\left(X_{n}\right)_{n \geq 1} \subset\{T\}^{\prime} \cap \mathcal{F}$ be such that $X_{n} x \rightarrow$ $X x$ for all $x \in \mathcal{H}$. For every $n \geq 1, X_{n} \mathcal{H}$ is a finite-dimensional subspace of $E_{T}$, invariant for $T$ and $T_{E}$. Therefore, $X_{n} \mathcal{H}$ is generated by some generalized eigenvectors of $T_{E}$. Thus $X_{n} \mathcal{H} \subset \operatorname{span} C\left(T_{E}\right)$ and $E_{T} \subset \operatorname{span} C\left(T_{E}\right)$.

Lemma 4.9. Let $T \in L(\mathcal{H})$ be a c.n.u. contraction such that $\mathbb{D} \backslash \sigma(T) \neq \emptyset$ and $I-T^{*} T \in S_{\infty}, T_{E}=\left.T\right|_{E_{T}}$. Then $\lambda \in \sigma_{\mathrm{p}}(T)$ if and only if $\lambda \in$ $\sigma_{\mathrm{p}}\left(T_{E}\right)$ and for all $\lambda \in \sigma_{\mathrm{p}}(T), C_{\lambda}(T)=C_{\lambda}\left(T_{E}\right)$. Therefore $\operatorname{span} C\left(T_{E}\right)=$ span $C(T)$.

Proof. If $\mathbb{D} \backslash \sigma(T) \neq \emptyset$ and $I-T^{*} T \in S_{\infty}$, then $\sigma(T) \cap \mathbb{D}=\sigma_{\mathrm{p}}(T)=$ $\sigma_{\mathrm{np}}(T)$ and for every $\lambda \in \sigma_{\mathrm{p}}(T)$, the Riesz projection $\mathcal{P}_{\lambda}$ is of finite rank and $C_{\lambda}(T)=\mathcal{P}_{\lambda} \mathcal{H}$. As $\mathcal{P}_{\lambda} \in\{T\}^{\prime}, C_{\lambda}(T) \subset E_{T}$ and $C_{\lambda}(T)=C_{\lambda}\left(T_{E}\right)$.

The following corollary is an obvious consequence of Lemmas 4.8 and 4.9 .

Corollary 4.10. Let $T \in L(\mathcal{H})$ be a c.n.u. contraction such that $\mathbb{D} \backslash$ $\sigma(T) \neq \emptyset$ and $I-T^{*} T \in S_{\infty}, T_{E}=\left.T\right|_{E_{T}}$. If $\{T\}^{\prime} \cap \mathcal{F}$ is SOT dense in $\{T\}^{\prime} \cap S_{\infty}$ then $E_{T}=\operatorname{span} C(T)$, that is, $E_{T}$ is generated by the generalized eigenvectors of $T$.

Recall that for a c.n.u. contraction $T \in L(\mathcal{H})$,

$$
\mathcal{H}_{0}(T)=\left\{x \in \mathcal{H}:\left\|T^{n} x\right\| \rightarrow 0\right\} .
$$


Recall also that if $T \in(\mathrm{SM})$ then the $C_{0}$. part $T_{0}=\left.T\right|_{\mathcal{H}_{0}(T)}$ of $T$ is in fact a $C_{0}$-contraction. Moreover $\Theta_{T_{0}}$ is the pure part of the inner factor $\Theta_{\text {inn }}$ of $\Theta_{T}$ and the minimal function $m_{T_{0}}$ of $T_{0}$ is the minimal scalar multiple of $\Theta_{\text {inn }}$.

Lemma 4.11. Let $T \in L(\mathcal{H})$ be an $(\mathrm{SM})$-contraction. Let $E_{T}$ and $\mathcal{H}_{0}(T)$ be defined by (10) and (11). Then $E_{T} \subset \mathcal{H}_{0}$. In particular $T_{E}=\left.T\right|_{E_{T}}$ is a $C_{0}$-contraction and $m_{T_{E}}$ divides $m_{T_{0}}$.

Proof. Let $X \in\{T\}^{\prime} \cap S_{\infty}$. We know that $T^{n}$ tends WOT to 0 . But $T^{n} X=X T^{n}$ and $X$ is compact, therefore $T^{n} X=X T^{n}$ tends SOT to 0 . Thus $X \mathcal{H} \subset \mathcal{H}_{0}(T)$. Consequently, $E_{T} \subset \mathcal{H}_{0}(T)$. As $E_{T}$ is invariant for $T$, it is invariant for $T_{0}$, thus $T_{E}=\left.T_{0}\right|_{E_{T}}$ is a $C_{0}$-contraction and $m\left(T_{E}\right)$ divides $m_{T}$ (see [SNF67, Proposition III.6.1]).

The following theorem is a completed version of the theorem given in Section 1.

THEOREM 4.12. Let $T \in L(\mathcal{H})$ be an (SM)-contraction such that $I-$ $T^{*} T \in S_{\infty}$. Set $\Theta=\Theta_{T}$, and let $\Theta=\Theta_{\text {out }} \Theta_{\text {inn }}$ be the outer-inner factorization of $\Theta$. Let $E_{T}$ and $\mathcal{H}_{0}(T)$ be defined by (10) and (11), and $T_{E}=\left.T\right|_{E_{T}}$. The following are equivalent:

(i) $\{T\}^{\prime} \cap \mathcal{F}$ is dense in $\{T\}^{\prime} \cap S_{\infty}$.

(ii) $\{T\}^{\prime} \cap \mathcal{F}$ is SOT dense in $\{T\}^{\prime} \cap S_{\infty}$.

(iii) $\left\{M_{\Theta_{\mathrm{inn}}}\right\}^{\prime} \cap \mathcal{F}$ is $(S O T)$ dense in $\left\{M_{\Theta_{\mathrm{inn}}}\right\}^{\prime} \cap S_{\infty}$.

(iv) $\Theta_{\text {inn }}$ has a Blaschke scalar multiple.

(v) $T_{0}=\left.T\right|_{\mathcal{H}_{0}}$ is complete.

(vi) The minimal function $m_{T_{E}}$ of $T_{E}$ is a Blaschke product.

Moreover, if (i)-(vi) are satisfied, then we have the following linear approximation:

$$
\lim _{n \rightarrow \infty}\left\|B_{n}(T) A-A\right\|=0, \quad \forall A \in\{T\}^{\prime} \cap S_{\infty},
$$

where $B=\prod_{k \geq 1} b_{\lambda_{k}}$ is the minimal scalar multiple of $\Theta_{\mathrm{inn}}, B_{n}=\prod_{k>n} b_{\lambda_{k}}$, and $B_{n}(T) A \in\{T\}^{\prime} \cap \mathcal{F}$ for all $n \geq 1$.

REMARK 4.13. (1) Let $T \in L(\mathcal{H})$ be a c.n.u. contraction such that $\mathbb{D} \backslash$ $\sigma(T) \neq \emptyset$ and $I-T^{*} T \in S_{\infty}$, and let $T_{0}$ be the $C_{0}$. part of $T$. Then $C(T)=C\left(T_{0}\right)=C\left(T_{E}\right)$, in other words the generalized eigenvectors of $T$, $T_{0}$ and $T_{E}$ are all the same. Indeed, if $\lambda \in \sigma(T)$, then the associated Riesz projection $\mathcal{P}_{\lambda}$ is in $\{T\}^{\prime} \cap \mathcal{F}$. Thus $C_{\lambda}(T)=\operatorname{Im} \mathcal{P}_{\lambda} \subset E$ and $C(\lambda) \subset E$. The fact that $E$ is invariant gives the conclusion.

(2) In the situation of Theorem 4.12 this means that $m_{T_{0}}$ and $m_{T_{E}}$ can differ within a singular inner factor only. If moreover $m_{T_{0}}$ is a Blaschke product, then so is $m_{T_{E}}$ (because $m_{T_{E}}$ divides $m_{T_{0}}$ ), therefore $m_{T_{E}}=m_{T_{0}}$ and $E_{T}=\operatorname{span} C\left(T_{E}\right)=\operatorname{span} C\left(T_{0}\right)=\mathcal{H}_{0}(T)$ (as a $C_{0}$-contraction having a Blaschke minimal function is complete), in other words $T_{0}=T_{E}$. 
(3) It is likely that the latter equality remains true even if $m_{T_{0}}$ is not a Blaschke product. Nevertheless we can only prove that $m_{T_{0}}=m_{T_{E}}$ for every (SM)-contraction with compact defect.

Proof of Theorem 4.12. We prove that (iv) $\Leftrightarrow(\mathrm{v}) \Rightarrow(\mathrm{vi}) \Rightarrow(\mathrm{i}) \Rightarrow$ (ii) $\Rightarrow$ (iv). The equivalence (iii) $\Leftrightarrow$ (iv) then follows from the equivalence (i) $\Leftrightarrow($ ii) $\Leftrightarrow$ (iv) applied to $M_{\Theta_{\text {inn }}}$.

The equivalence $(\mathrm{v}) \Leftrightarrow(\mathrm{iv})$ is clear because a $C_{0}$-contraction is complete if and only if its minimal function is a Blaschke product, and the minimal function of $T_{0}$ is the minimal scalar multiple of $\Theta_{\text {inn }}$.

We shall see now that $(\mathrm{vi}) \Rightarrow(\mathrm{i})$. Let $m_{T_{E}}=B=\prod_{k \geq 1} b_{\lambda_{k}}$ and $B_{n}=$ $\prod_{k>n} b_{\lambda_{k}}, n \geq 1$. As $\left|B_{n}(\xi)\right|=1$ a.e. on $\mathbb{T}$ we have

$$
\begin{aligned}
\int_{\mathbb{T}}\left|B_{n}(\xi)-1\right|^{2} d \mu(\xi) & =\int_{\mathbb{T}}\left|B_{n}(\xi)\right|^{2} d \mu(\xi)+1-2 \Re\left(\int_{\mathbb{T}} B_{n}(\xi) d \mu(\xi)\right) \\
& =2\left(1-B_{n}(0)\right) \rightarrow 0 .
\end{aligned}
$$

This implies that $\left\|B_{n}(T) x-x\right\| \rightarrow 0$ for every $x \in E_{T}$, so $\left\|B_{n}(T) X-X\right\|=$ $\left\|X B_{n}(T)-X\right\| \rightarrow 0$ for every $X \in\{T\}^{\prime} \cap S_{\infty}$. But $B_{n}(T) X \in\{T\}^{\prime} \cap \mathcal{F}$ as

$$
B_{n}(T) X \mathcal{H} \subset \operatorname{ker} \frac{B}{B_{n}}(T) \subset \operatorname{span}\left\{C_{\lambda_{k}}(T): 1 \leq k<n\right\}
$$

and each $C_{\lambda_{k}}\left(T_{E}\right)$ is finite-dimensional (all eigenvalues are of finite type). This also proves the last assertion of the theorem.

(i) $\Rightarrow$ (ii) is obvious. Now, we show that (ii) $\Rightarrow$ (iv). Suppose (ii) and suppose that the minimal scalar multiple of $\Theta$ has a non-trivial singular part. We shall work with the model $M_{\Theta}$ of $T$ to show that this leads to a contradiction. We know that if $A \in\{T\}^{\prime} \cap \mathcal{F}$, then $A \mathcal{H}$ is a finite-dimensional invariant subspace of $T$, and hence $A \mathcal{H} \subset C(T)$ and $B(T) A=0$, where $C(T)$ is defined according to (9). Condition (ii) implies that $B\left(M_{\Theta}\right) A=0$ for every $A \in\left\{M_{\Theta}\right\}^{\prime} \cap S_{\infty}$.

Let $\delta$ be a minimal scalar multiple of $\Theta$. Assuming that $\delta$ contains a nontrivial singular inner factor we obtain a contradiction to the above property of $B\left(M_{\Theta}\right)$. Let $m_{1}$ be a non-trivial singular factor of $\delta$ such that $\sigma\left(m_{1}\right)$ has Lebesgue measure zero, and $f \in \mathcal{C}_{A}$ an outer function equal to zero on $\sigma\left(m_{1}\right)$. According to Lemma 3.7, if we set $\psi=f \delta / m_{1}$ then $H_{\psi \Theta^{-1}}$ is compact. Then, Corollary 4.5 says that the operator $X=\left.\left(\begin{array}{c}\Theta \\ \Delta\end{array}\right) P_{-} \Theta^{-1} \psi P_{*}\right|_{K_{\Theta}}$ is in $\left\{M_{\Theta}\right\}^{\prime} \cap S_{\infty}$. Suppose $\psi \Theta^{-1}=G \in H^{\infty}\left(L\left(\mathcal{D}_{T^{*}}, \mathcal{D}_{T}\right)\right)$, then

$$
\psi I=f \frac{\delta}{m_{1}} I=\Theta G,
$$

and hence $f \delta / m_{1}$ is a scalar multiple of $\Theta_{\text {inn }}$, which is impossible since $\delta$ is a minimal scalar multiple. Therefore, $\psi \Theta^{-1} \notin H^{\infty}\left(L\left(\mathcal{D}_{T^{*}}, \mathcal{D}_{T}\right)\right)$. 
Now, consider $B\left(M_{\Theta}\right) X$. As

$$
Y=\left(\begin{array}{cc}
\psi I & 0 \\
\psi \Delta \Theta^{-1} & 0
\end{array}\right)
$$

is a lifting of $X$ (see the proof of Lemma 4.4), a lifting of $B\left(M_{\Theta}\right) X$ is

$$
\left(\begin{array}{cc}
B \psi & 0 \\
B \psi \Delta \Theta^{-1} & 0
\end{array}\right)
$$

Thus

$$
B\left(M_{\Theta}\right) X=\left.\left(\begin{array}{c}
\Theta \\
\Delta
\end{array}\right) P_{-} \Theta^{-1} B \psi P_{*}\right|_{K_{\Theta}}
$$

Clearly, $B \psi \Theta^{-1}$ is still in $H^{\infty}\left(L\left(\mathcal{D}_{T^{*}}, \mathcal{D}_{T}\right)\right)+\mathcal{C}\left(\mathbb{T}, S_{\infty}\left(\mathcal{D}_{T^{*}}, \mathcal{D}_{T}\right)\right)$ but is not in $H^{\infty}\left(L\left(\mathcal{D}_{T^{*}}, \mathcal{D}_{T}\right)\right)$ by the same minimality reason as above. Thus $B\left(M_{\Theta}\right) X$ is a non-zero operator. This gives a contradiction to the above property of $B\left(M_{\Theta}\right)$.

5. Schatten-von Neumann operators in the commutant. The following theorem contains some sufficient conditions for $\{T\}^{\prime} \cap S_{p} \neq\{0\}$. The meaning of these conditions is that there exist some part of the operator $T$ which has "thin" spectrum. To express this "thinness" we make use of the notions of maximal spectral subspace and of Beurling-Carleson subsets of the unit circle. Recall that, for a compact set $\sigma \subset \mathbb{C}$, the maximal spectral subspace $H(\sigma)$ over $\sigma$ is defined by the following requirements:

(i) $H(\sigma) \subset \mathcal{H}$ is an invariant subspace for $T$ such that $\sigma\left(\left.T\right|_{H(\sigma)}\right) \subset \sigma$;

(ii) if $E$ is another $T$-invariant subspace with $\sigma\left(\left.T\right|_{E}\right) \subset \sigma$ then $E \subset$ $H(\sigma)$.

It is known that for c.n.u. contractions of the class (SM), the maximal spectral subspaces $H(\sigma)$ exist for all closed sets $\sigma \subset \overline{\mathbb{D}}$ (see [SNF67]). Moreover, if $\delta$ is a minimal scalar multiple of $\Theta_{T}$ and $\sigma \cap \mathbb{T}$ is a subset of Lebesgue measure 0 , then $H(\sigma) \neq\{0\}$ if and only if $\delta_{\sigma} \neq 1$, where

$$
\delta_{\sigma}(z)=\left(\prod_{\lambda_{n} \in \sigma \cap \mathbb{D}} b_{\lambda_{n}}(z)\right) \exp \left(-\int_{\sigma \cap \mathbb{T}} \frac{\xi+z}{\xi-z} d \mu_{\delta}(\xi)\right),
$$

and

$$
\delta=\left(\prod_{n \geq 1} b_{\lambda_{n}}(z)\right) \exp \left(-\int_{\mathbb{T}} \frac{\xi+z}{\xi-z} d \mu_{\delta}(\xi)\right)
$$

is the canonical factorization of $\delta$ (the measure $\mu_{\delta}=\log (1 /|\delta|) d \mu+\mu_{\delta}^{\mathrm{s}}$ contains both absolutely continuous and singular parts); here $\mu$ denotes the Lebesgue measure on $\mathbb{T}$. Therefore, for a subset $\sigma \subset \mathbb{T}$ of Lebesgue measure zero, $H(\sigma) \neq\{0\}$ if and only if $\mu_{\delta}^{\mathrm{s}}(\sigma)>0$. 
Recall also that a closed subset $\sigma \subset \mathbb{T}$ is called a Beurling-Carleson set if

$$
\int_{\mathbb{T}} \log (\operatorname{dist}(\xi, \sigma)) d \mu(\xi)>-\infty,
$$

or equivalently, if $\mu(\sigma)=0$ and $\sum_{k>1}\left|I_{k}\right| \log \left|I_{k}\right|^{-1}<\infty$, where $\left(I_{k}\right)_{k \geq 1}$ is the sequence of complementary arcs of $\sigma$. For more information on BeurlingCarleson sets see, for example, V. Havin and B. Jöricke [HJ94, Section II.3.1].

TheOREM 5.1. Let $T$ be an (SM)-contraction such that $I-T^{*} T \in S_{p}$ with $1 \leq p<\infty$. Then $\{T\}^{\prime} \cap S_{p} \neq\{0\}$ as soon as one of the following properties is satisfied:

(i) $\sigma_{\mathrm{p}}(T) \neq \emptyset$, or equivalently $\operatorname{ker} \Theta(\lambda) \neq\{0\}$ for some $\lambda \in \mathbb{D}$.

(ii) There exists a Beurling-Carleson set $\sigma \subset \mathbb{T}$ such that $H(\sigma) \neq\{0\}$. Moreover if $T \in C_{0}$ then $H^{\infty}(T) \cap S_{p} \neq\{0\}$.

Before starting the proof, we give modified versions of Lemmas 3.6 and 3.7 for the $S_{p}$ classes.

Lemma 5.2. Let $T \in L(\mathcal{H})$ be a $C_{0}$-contraction such that $I-T^{*} T \in S_{p}$ $(1 \leq p<\infty)$ and $m_{T}$ the minimal function of $T$. Assume that there exists a Beurling-Carleson set $\sigma \subset \mathbb{T}$ such that $\left(m_{T}\right)_{\sigma}$ is non-constant. Then there exists $\varphi \in H^{\infty}$ such that $\varphi(T) \neq 0$ and the Hankel operator $H_{\varphi \Theta_{T}^{*}}$ belongs to $S_{p}$. More precisely, we can take $\varphi=f m_{T} / m_{1}$, where $m_{1}=\left(m_{T}\right)_{\sigma}$ and $f$ is any outer $\mathcal{C}_{A}^{\infty}$ function equal to zero on $\sigma$.

For the proof the following characterization of Hankel operators from the class $S_{p}$ in terms of their symbols is used. Let $\Phi \in L^{\infty}\left(L\left(\mathcal{H}_{1}, \mathcal{H}_{2}\right)\right)$ and $1 \leq p<\infty$. Then $H_{\Phi} \in S_{p}$ if and only if $P_{-} \Phi \in B_{p}^{1 / p}\left(S_{p}\right)$, where $B_{p}^{1 / p}\left(S_{p}\right)$ denotes the Besov class with values in $S_{p}$ (see [Pel82]).

Proof of Lemma 5.2. The proof is similar to that of Lemma 3.6. The only difference is that $f$ can be chosen to be an outer function in $\mathcal{C}_{A}^{\infty}=$ $\mathcal{C}_{A} \cap \mathcal{C}^{\infty}$ such that $|f(\xi)|=o\left((\operatorname{dist}(\xi, \sigma))^{N}\right)$ as $\xi$ tends to $\sigma$, for every $N \geq 1$ (see [HJ94, Section II.3.1] for the existence of such a function). Here $\mathcal{C}^{\infty}$ stands for the space of infinitely differentiable functions on $\mathbb{T}$. We denote by $\mathcal{C}^{\infty}(X)$ the space of $X$-valued $\mathcal{C}^{\infty}$ functions. Then, in the notation of Lemma 3.6, $f \Theta_{1}(z)^{*}=f A^{*}+f K(z)^{*}$, where $f \Theta_{1}^{*} \in \mathcal{C}^{\infty}\left(L\left(\mathcal{K}, \mathcal{D}_{T}\right)\right)$ and consequently $K \in \mathcal{C}^{\infty}\left(S_{p}\left(\mathcal{D}_{T}, \mathcal{K}\right)\right)$. On the other hand, it is easy to see that $\mathcal{C}^{\infty}(X) \subset B_{p}^{1 / p}(X)$ for any Banach space $X$ and any $0<p<\infty$. Therefore, by Peller's theorem the Hankel operator $H_{f \Theta_{1}^{*}}$ is in $S_{p}$, and the same holds for $H_{f \Theta^{*}}$.

Lemma 5.3. Let $T \in(\mathrm{SM}), T \notin C_{11}$, be such that $I-T^{*} T \in S_{p}, 1 \leq p$ $<\infty$, and let $\delta$ be a minimal scalar multiple of $T$. Assume that there exists 
a Beurling-Carleson set $\sigma \subset \mathbb{T}$ such that $\delta_{\sigma}$ is non-constant. Then there exists $\psi \in H^{\infty}$ such that $H_{\psi \Theta_{T}^{-1}}$ is in $S_{p}$ but $\psi \Theta_{T}^{-1} \notin H^{\infty}\left(L\left(\mathcal{D}_{T^{*}}, \mathcal{D}_{T}\right)\right)$. More precisely, we can take $\psi=f \delta / m_{1}$, where $m_{1}=\left(\delta_{\text {inn }}\right)_{\sigma}, \delta_{\text {inn }}$ being the inner part of $\delta$, and $f$ is any outer $\mathcal{C}_{A}^{\infty}$ function equal to zero on $\sigma$.

Proof. The proof follows that of Lemma 3.7 with the obvious modifications, in particular Lemma 3.6 is replaced by Lemma 5.2.

Proof of Theorem 5.1. If (i) is satisfied, then the Riesz projection $\mathcal{P}_{\lambda}$ belongs to $\{T\}^{\prime} \cap \mathcal{F}$. If (ii) is satisfied, identify $T$ with its model operator $M_{\Theta}$ and define $X$ by

$$
X=\left.\left(\begin{array}{c}
\Theta \\
\Delta
\end{array}\right) P_{-} \Theta^{-1} \psi P_{*}\right|_{K_{\Theta}},
$$

where $\psi$ is defined as in Lemma 5.3. Then, as in the proof of Theorem 4.7, $X$ is a compact operator in $\{T\}^{\prime}$ which is also non-zero and in $S_{p}$ due to Lemma 5.3.

In the case when $\Theta$ is a scalar $H^{\infty}$ function, Theorem 5.1 extends to all $p$, $0<p<\infty$, as the characterization of Hankel operators of class $S_{p}$ is valid for $0<p<\infty$ in the case when the symbols are scalar-valued (see [Pel83]).

Acknowledgments. The results above are mostly contained in the $\mathrm{PhD}$ thesis written by the author during her studies at the University of Bordeaux 1. The author is very grateful to Professor N. K. Nikolski for useful suggestions and friendly encouragements.

\section{References}

[Ber88] H. Bercovici, Operator Theory and Arithmetic in $H^{\infty}$, Amer. Math. Soc., Providence, RI, 1988.

[BP75] F. F. Bonsall and S. C. Power, A proof of Hartman's theorem on compact Hankel operators, Math. Proc. Cambridge Philos. Soc. 78 (1975), 447-450.

[GK69] I. C. Gohberg and M. G. Krein, Introduction to the Theory of Linear Nonselfadjoint Operators, Amer. Math. Soc., Providence, RI, 1969.

[HJ94] V. Havin and B. Jöricke, The Uncertainty Principle in Harmonic Analysis, Springer, Berlin, 1994.

[MN75] B. Moore III and E. A. Nordgren, On transitive algebras containing $C_{0}$ operators, Indiana Univ. Math. J. 24 (1974/75), 777-784.

[Muh69] P. S. Muhly, Commutants containing a compact operator, Bull. Amer. Math. Soc. 75 (1969), 353-356.

[Muh71] -, Compact operators in the commutant of a contraction, J. Funct. Anal. 8 (1971), 197-224.

[Nik71] N. K. Nikolski, Five problems on invariant subspaces, Zap. Nauchn. Sem. Leningrad. Otdel. Mat. Inst. Steklov. (LOMI) 23 (1971), 115-127.

[Nik86] -, Treatise on the Shift Operator, Springer, Heidelberg, 1986. 
[NV98] N. K. Nikolski and V. I. Vasyunin, Elements of spectral theory in terms of the free function model. I. Basic constructions, in: Holomorphic Spaces (Berkeley, CA, 1995), Cambridge Univ. Press, 1998, 211-302.

[Nor75] E. A. Nordgren, Compact operators in the algebra generated by essentially unitary $C_{0}$ operators, Proc. Amer. Math. Soc. 51 (1975), 159-162.

[Pel82] V. V. Peller, Vectorial Hankel operators, commutators and related operators of the Schatten-von Neumann class $\mathfrak{S}_{p}$, Integral Equations Operator Theory 5 (1982), 244-272.

[Pel83] - Description of Hankel operators of the class $\mathfrak{S}_{p}$ for $p>0$, investigation of the rate of rational approximation and other applications, Mat. Sb. (N.S.) 122 (164) (1983), 481-510 (in Russian); English transl.: Math. USSR-Sb. 50 (1985), 465-494.

[Sar67] D. Sarason, Generalized interpolation in $H^{\infty}$, Trans. Amer. Math. Soc. 127 (1967), 179-203.

[SN74] B. Sz.-Nagy, On a property of operators of class $C^{0}$, Acta Sci. Math. (Szeged) 36 (1974), 219-220.

[SNF67] B. Sz.-Nagy et C. Foiaş, Analyse harmonique des opérateurs de l'espace de Hilbert, Masson, 1967.

Department of Mathematics and Statistics

Laval University

Québec, Canada G1K 7P4

E-mail: pvitse@mat.ulaval.ca

Received October 1, 2001

Revised version June 13, 2002 